Deposition on flexible substrates
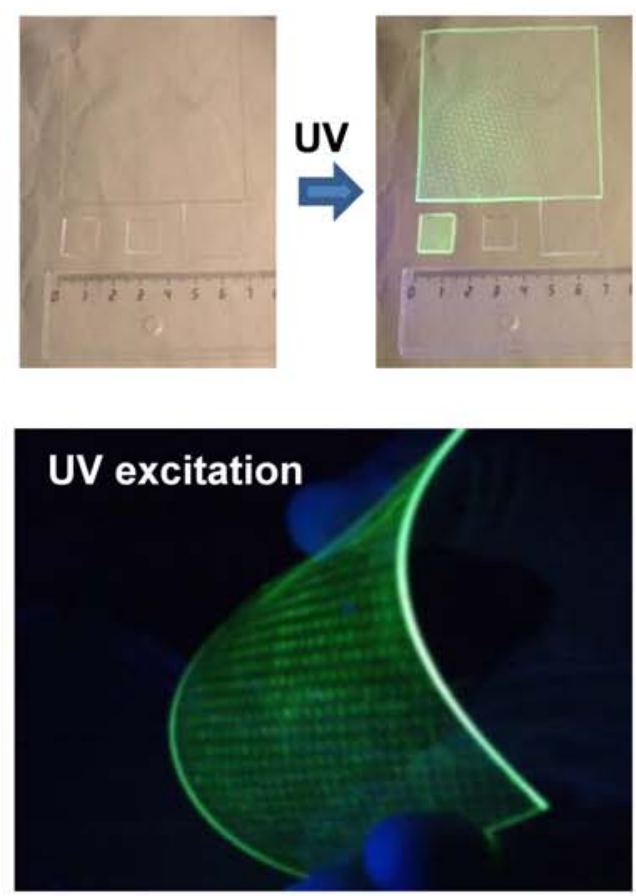

Adjustable refractive index

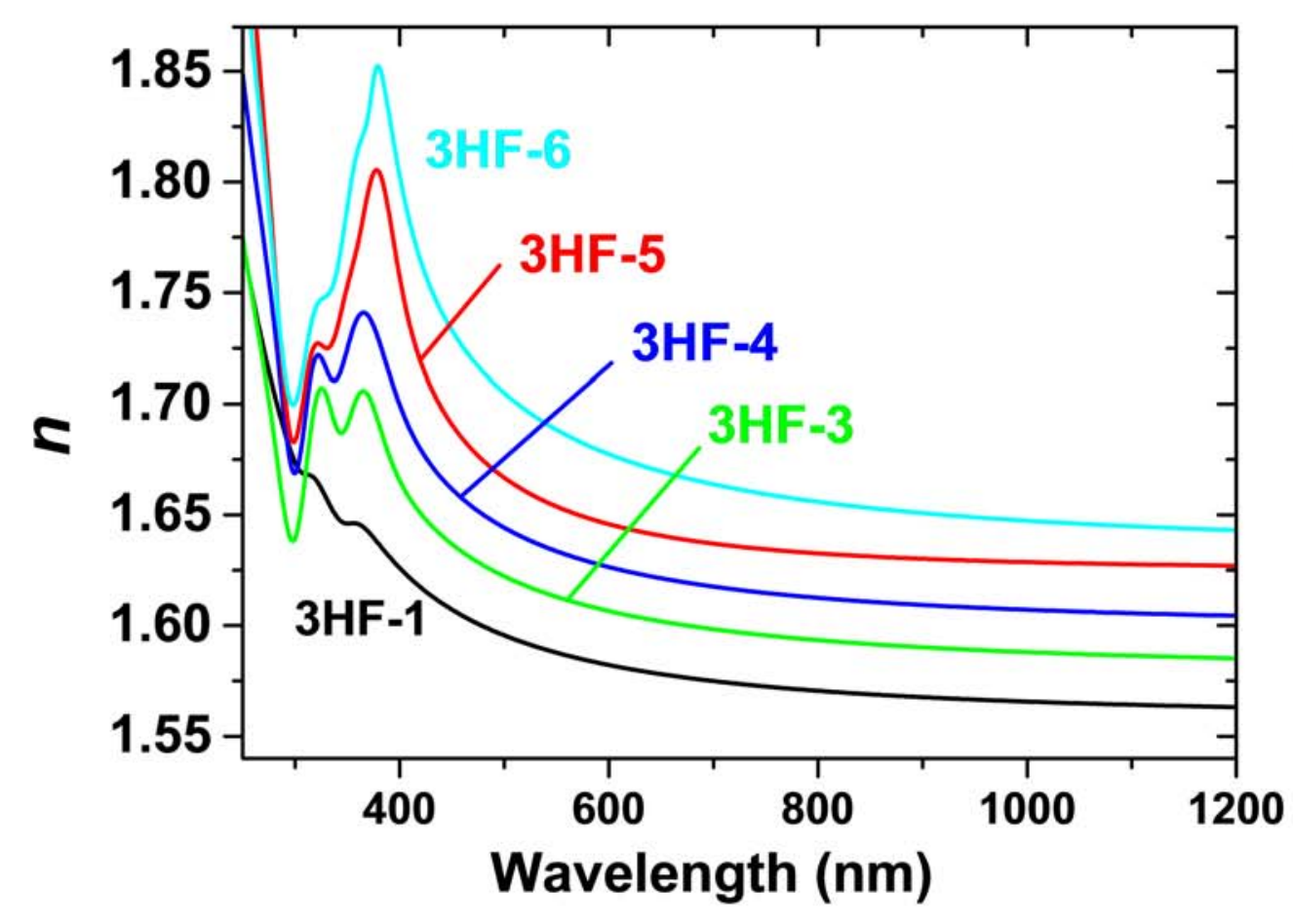

Luminescent UV Detection

UV
irradiation Emission

$57 \mu \mathrm{W} \mathrm{cm}-2$

$33 \mu \mathrm{W} \mathrm{cm}-2$

$13 \mu \mathrm{W} \mathrm{cm}-2$

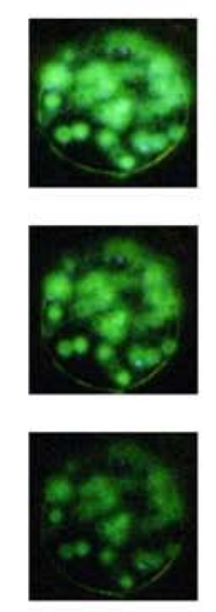




\title{
Luminescent 3-Hydroxyflavone nanocomposites with tuneable refractive index for photonics and UV detection by plasma assisted vacuum deposition
}

Francisco J. Aparicio ${ }^{\mathrm{a}}$, María Alcaire ${ }^{\mathrm{a}}$, Ana Borras ${ }^{\mathrm{a}}$, Juan C. Gonzalez ${ }^{\mathrm{a}}$, Fernando LópezArbeloa ${ }^{\mathrm{b}}$, Iwona Blasczcyk-Lezak ${ }^{\mathrm{a}}$, Agustín R. González-Elipe ${ }^{\mathrm{a}}$ and Angel Barranco ${ }^{{ }^{*}}$

\footnotetext{
${ }^{a}$ Consejo Superior de Investigaciones Científicas. Instituto de Ciencia de Materiales de Sevilla (CSIC-Universidad de SevillaJunta de Andalucía), c/Américo Vespucio 49, 41092 Sevilla, Spain. Email fjaparicio@icmse.csic.es (F.J. Aparicio) and angel.barranco@csic.es (A. Barranco).

${ }^{b}$ Departamento de Química Física. Universidad del País Vaco UPV/EHU, Apartado 644, 48080-Bilbao, Spain
}

\begin{abstract}
Luminescent organic-thin-films transparent in the visible region have been synthetized by a plasma assisted vacuum deposition method. The films have been developed for their implementation in photonic devices and for UV detection. They consist in a plasma polymeric matrix that incorporates 3-hydroxyflavone molecules characterized by absorbing UV radiation and emitting green light. The present work studies in detail the properties and synthesis of this kind of transparent and luminescent materials. The samples have been characterized by X-ray photoemission (XPS), infrared (FT-IR) and secondary ion mass (ToFSIMS) spectroscopies; and their optical properties analysed by UV-Vis absorption, fluorescence and ellipsometry (VASE) spectroscopies. The key factors controlling the optical and luminescent properties of the films are also discussed. Indeed, our experimental results show how the optical properties of the films can be adjusted for their integration in photonic devices. Moreover, time resolved and steady state fluorescence analyses, including quantum yield determination, indicate that the fluorescence efficiency is a function of the deposition parameters. An outstanding property of these materials is that, even for high UV absorption values (i.e. large layer thickness and/or dye concentration), the emitted light is not reabsorbed by the film. Such a highly UV absorbent and green emitting films can be used as UV photodetectors with a detection threshold smaller than $10 \mu \mathrm{W} \mathrm{cm}{ }^{-2}$, a value similar to the limit of some commercial UV photodetectors. Based on these properties, it is also proposed the use of the films as visual tags for the detection of solar UV irradiation.
\end{abstract}

\section{INTRODUCTION}

Dye based luminescent materials have been used as active media in optoelectronic devices, ${ }^{1}$ solar cells, ${ }^{2}$ laser cavities, ${ }^{3}$ and photonic structures, in this latter case with promising applications in optical communications ${ }^{4}$ and information processing. ${ }^{5}$ This kind of luminescent films are usually synthesized by vacuum sublimation and wet routes such as sol-gel and chemical polymerization in solution. However, such methods present some limitations when aiming at the integration of the films in photonic and optoelectronic devices. This fosters the development of solvent-less procedures for the synthesis of robust luminescent thin films. Recently, we have reported the fabrication of different luminescent photonic devices by the integration of a new kind of 3hydroxyflavone (3-HF) fluorescent films in photonic resonant structures (i.e., vertical 
cavities as well as waveguide integrated microdisk and Fabry-Perot resonators). ${ }^{6}$ The films were synthetized by a plasma assisted vacuum deposition (PAVD) technique developed during the last years in our laboratory for the deposition of dye containing plasma polymer films. ${ }^{6-13}$

In a conventional plasma polymerization process, a vapour pressure of an organic precursor is dosed into a plasma discharge for the synthesis of a solid thin film. This is a complex process that involves the breaking of the starting monomer and different chemical reactions between the resulting species (both reactive and stable groups), as well as, further fragmentation and reorganization processes. ${ }^{14-18}$ Plasma polymers consist in highly cross-linked organic films formed by the plasma fragmentation and recombination of the precursor molecules. In general, this kind of highly branched films do not retain the original bond structures or the functional groups present in the starting precursor and their properties are mainly defined by the deposition conditions. Hence, retention of the precursor functionalities in the growing film is always a challenge for the plasma polymerization technique. This question has been addressed by several researchers by making use of pulse discharges, high pressures and other experimental approaches. ${ }^{19-22}$ Such kind of functional plasma polymers synthetized at low or atmospheric pressure have been mainly developed for biomaterials and medical applications. ${ }^{23,24}$ Plasma polymerization and plasma treatment processes are also used for the development of organic-inorganic nanocomposites for electrical insulation, ${ }^{25}$ optical coatings, ${ }^{26}$ and thin films with potential optoelectronics applications. ${ }^{27}$ The PAVD process employed in this work makes use of a remote plasma discharge in combination with the low pressure sublimation of an organic solid monomer over the substrates located in the afterglow. As an advance with respect to conventional plasma polymerization, this approach allows to grow a plasma polymer matrix retaining a substantial amount of integer photofunctional precursor molecules which have not been fragmented by the discharge during the film deposition. This method have been successfully applied for the development of photonic layers ${ }^{6,7}$ with tailored luminescent ${ }^{10}$ and optical properties, ${ }^{8}$ as well as for the development of photonic gas sensors. ${ }^{9,11}$ This is done by finely regulating of the interaction between the low power microwave plasma and the precursor molecules sublimated in the afterglow region. Such a control requires simultaneously adjusting different deposition parameters. The most important are the $\mathrm{MW}$ power, the total pressure, the sublimation rate and the geometrical arrangement used for the deposition. Previous investigations demonstrated that the distance between the plasma and the growing film $(z)$ is one of the key working parameter controlling the interaction between the discharge and the precursor molecules involved in the growing of the PAVD films. ${ }^{9-11}$ Therefore, this distance gradually regulates the film properties: from a highly cross-linked plasma polymer that do not retain the precursor structure (deposition at the glow discharge) to plasma films containing a controllable amount of luminescent active dye molecules (deposition in the afterglow). ${ }^{9-11}$ If the distance to the glow discharge is further increased, the films are similar to those obtained by vacuum deposition in absence of a plasma. ${ }^{11}$ As a novelty in the present research the influence of the deposition rate (controlled by the sublimation speed) is also investigated.

The present work reports about the synthesis and properties of 3-HF plasma polymer thin films deposited by this approach. 3-Hydroxyflavone is one of the most widely studied flavonol derivatives because it may exhibit a dual fluorescence (blue and green bands) under UV illumination. The blue emission is ascribed to the normal form of the 3-HF. Whereas the large Stokes shifted emission in the green region is assigned to the tautomer generated through an excited state intermolecular proton transfer (ESIPT) process. ${ }^{28,29}$ The ESIPT mechanism of $3-\mathrm{HF}$ was initially described by Sengupta and 
Kasha $^{30}$ and further studied by many authors during the last decades. ${ }^{31-33}$ This process involves a very efficient and non-radiative energy dissipating mechanism ${ }^{34}$ whereby the hydroxyl proton of the molecule is transferred to a neighbouring carbonyl group when the molecules is in the excited state. The proton transfer process is very sensitive to the chemical environment surrounding the 3-HF molecules, particularly to the formation probability of $\mathrm{H}$ bonds with nearby molecules or groups. Thus, the relative intensity of the blue and green emission bands is controlled by the hydrogen bond donor ability of the environment. ${ }^{30-32,35}$ Due to its singular luminescent properties the 3-HF has been used in the development of dye lasers, scintillator, wavelength shifters, UV detectors in commercial Si photodiodes, etc. ${ }^{36-38}$

The first purpose of the present work is to study in detail the synthesis, composition, and structural characteristics of the 3-HF PAVD films. Secondly it is shown how the synthetic procedure enables to adjust the optical properties (extinction coefficient and refractive index) of the films for their implementation as active media in luminescent photonic devices. ${ }^{6}$ Intending to improve the luminescent properties, the influence of the plasma polymeric matrix on the quantum yield of the trapped 3-HF molecules is also investigated. Finally we explore the use the films as UV photodetectors. Most common UV photodetectors make use of wide band gap inorganic semiconductors ${ }^{39,40}$ or organic photodiodes. ${ }^{41}$ The alternative procedure used here to detect UV photons consists in the development of a dye coating that under UV irradiation presents a strong green emission in the centre of the visible spectrum. This kind of films deposited onto visible photodetectors can be used to extend their spectral response to the UV region. ${ }^{38}$ Depending on their emission efficiencies, the samples can be also employed for the development of visual tags for UV detection.

\section{EXPERIMENTAL AND METHODS}

\subsection{Materials}

3-Hydroxyflavone powders were purchased from Sigma-Aldrich and used as received without further purifications. UV transparent fused silica slides, as well as, doped and intrinsic $\mathrm{Si}(100)$ pieces (both $1 \times 1 \mathrm{~cm}^{2}$ of area) were used as substrates.

\subsection{Plasma assisted vacuum deposition procedure}

A complete description of the experimental setup used for the deposition can be found elsewhere. ${ }^{11,42}$ This consists in an electron cyclotron resonance (ECR) plasma reactor with two zones for the plasma and for the remote deposition. In the plasma zone, an Argon microwave plasma (power $150 \mathrm{~W}$ and pressure $10^{-2} \mathrm{mbar}$ ) is sustained and confined thanks to a set of magnets. Meanwhile, a set of homemade Knudsen cells located in the deposition zone sublimate the dye onto the substrates also placed in the afterglow of the plasma discharge. For the synthesis of the films two different geometrical arrangements were used (see Fig. S1 in the supporting information section). In both configurations the sample holder consists in a grounded metallic mesh ( $1 \mathrm{~mm}$ of thickness) placed between the Knudsen cells and the plasma zone. In the case of the back configuration the substrates are fixed to the bottom face of the sample holder, facing the sublimation cells. Whereas in the front configuration they are mounted onto the top face, and directly exposed to the plasma discharge. For both arrangements the distance $z$ between sample holder and plasma zone is a critical parameter that permits to regulate the interaction between the sublimated dye and the plasma species, and therefore the properties of the final deposit. ${ }^{9-11,13}$ Film thickness and deposition rate $(r)$ were monitored by using a quartz crystal microbalance (QCM) besides the sample holder. The registered values were corrected by a calibration factor 
obtained from the thickness of the films determined after deposition. The amount of precursor molecules dosed into the deposition zone, and therefore the deposition rate, were regulated by heating the sublimation cells. In our evaporation system the temperature is measured in the external shell of a homemade Knudsen cell. Note that this reading does not provide a reliable measurement of the actual temperature inside the cell during the sublimation. Thus, the precursor supply was controlled by the QMC by slightly modifying the Knudsen-cell heating during the deposition. This procedure is by far more reproducible that the direct control of the cell temperature.

A set of 3-HF plasma polymer thin films ( $175 \mathrm{~nm}$ of thickness) were grown by varying the deposition conditions as summarized in Table 1 . The main goal of this study was the synthesis of plasma polymeric like films with a controllable amount of entire and luminescent active 3-HF molecules. This was accomplished by adjusting different deposition parameters in order to regulate the fragmentation of precursor molecules during the deposition and the incorporation of embedded dye molecules in the growing film. As aforementioned for a given plasma conditions (i.e., power, pressure of gas, etc.) increasing the distance $z$ between the substrates and the discharge is an efficient way to increase the concentration of integer dye molecules in the films. ${ }^{9-11}$ The second working parameter was the deposition rate. It is expected that, for fixed plasma conditions, the percentage of dye molecules remaining intact during the deposition augments with the deposition rate that is proportional to the sublimation rate. Thus, experimental conditions in Table 1 were selected intending to gradually increase the concentration of integer dye molecules from sample $3 \mathrm{HF}-1$ to $3 \mathrm{HF}-2$ and from sample $3 \mathrm{HF}-3$ to $3 \mathrm{HF}-6$. Other parameters such as the plasma power and the working pressure also provide a way to control the films characteristics. It is interesting to indicate that the range of plasma parameters studied in this work is mainly limited to the final application of the synthesized films as optical/luminescent materials. In this sense, our results indicate the plasma power have to be limited to a few hundred watts (depending on the precursor) in order to avoid the fragmentation of all the precursor molecules. On the other hand, increasing the working pressure may leads to the formation of dye aggregates, as reported for other organic dyes. ${ }^{10}$ In the present study, we preferred to regulate the geometrical arrangement and the deposition rate as working parameters because they rendered more reproducible and controllable results. A plasma power of $150 \mathrm{~W}$ and a working pressure of $10^{-2}$ mbar were chosen in order to obtain a higher variation on the films properties as a function of the selected working parameters keeping the optical quality of the deposit.

\subsection{Thin film characterization}

The surface chemical composition of the samples was analysed by X-ray photoelectron spectroscopy (XPS) using an ESCALAB 210 spectrometer operating at constant pass energy of $20 \mathrm{eV}$. Nonmonochromatized $\mathrm{Mg} \mathrm{K \alpha}$ radiation was used as excitation source. The atomic surface concentrations were quantitatively determined from the area of C1s and $01 \mathrm{~s}$ peaks. A Shirley-type background was subtracted, and the peak areas were corrected by the electron escape depth, the spectrometer transmission and the photoelectron cross sections. ${ }^{43}$ The molecular structure of the samples was studied by time-of-flight secondary-ion mass spectrometry (TOF-SIMS) using an instrument from Ion-ToF GmbH Germany. The samples were bombarded with a pulsed bismuth ion beam. The generated secondary ions were extracted with a $10 \mathrm{kV}$ voltage and their time of flight, from the sample to the detector, was measured in a reflectron mass spectrometer. Typical analysis conditions in this work were $25 \mathrm{keV}$ pulsed $\mathrm{Bi}^{+}$ion beam at $45^{\circ}$ incidence rastered over $500 \times 500 \mathrm{\mu m}^{2}$. An electron flood gun for charge compensation was used during the measurements. The peak intensities were 
normalized to the total ion intensity. Fourier transform infrared (FT-IR) spectra were recorded in a Jasco 6200 spectrometer operating in transmission mode for thin-films samples deposited on $\mathrm{Si}(100)$ wafers and powder samples in $\mathrm{KBr}$ pellets.

The surface topography of the films was imagined by noncontact atomic force microscopy (AFM) with a Cervantes AFM system from NANOTEC and using commercial noncontact AFM tips from MikroMasch.

Thermal stability of the films was analysed in a tubular oven in vacuum $\left(10^{-2}-10^{-3}\right.$ mbar) by heating the samples at $150{ }^{\circ} \mathrm{C}$ during $1 \mathrm{~h}$. Solubility tests were conducted in water and toluene, for a period of $2 \mathrm{~h}$. After each immersion test, the films were dried in flowing nitrogen during $1 \mathrm{~h}$.

UV-Vis transmission spectra were acquired in a Varian Cary 300 spectrophotometer. The optical properties of the thin films were also studied with a variable angle spectroscopic ellipsometer (VASE) from J.A. Woollam Co., Inc. Depolarization measurements were performed at three incidence angles: $60^{\circ}, 65^{\circ}$, and $70^{\circ}$. Transmission and depolarization data were analysed assuming the Cauchy dispersion law and an ensemble of three Lorentz oscillators. The thickness of the films were measured with a Mahr Surf XR-20 profilometer and confirmed by ellipsometry.

Fluorescence spectra were recorded in a Horiba Jobin-Yvon FluoroLog 3 spectrofluorometer operating in the front face mode. The emission spectra were excited with radiation of $350 \mathrm{~nm}$ and 0.4 and $3 \mathrm{~nm}$ slits were used for the excitation and emission monochromators, respectively. The external radiative quantum efficiency of the films $(\eta),{ }^{44}$ defined as the ratio between the number of emitted photons and the number of photons absorbed by the film, was determined by an absolute method. The used set up consists in an integrating sphere Labsphere ${ }^{45}$ with its inner face coated with Spectralon and attached to the spectrofluorometer. Spectral correction curves for the sphere reflectance and the emission detector were provided by Horiba Jobin-Yvon. In the analysed samples the dye absorption band overlaps with the UV absorption of the plasma polymeric matrix, as it is shown in section 3.2. Since $\eta$ is calculated from the absorption of the whole system (luminescent molecules and the inactive polymeric matrix), it does not correspond to the dye quantum yield. Hence the quantum yield (Ф) of the green emission of the 3-HF molecules embedded in the films has to be calculated by applying a correction factor corresponding to the percentage of light absorbed by the active dye molecules in relation to the absorption of the whole sample. This is done by Equation 1 (see supporting information Section S2), where $A_{\text {film }}$ is the absorbance of the sample and $A_{\text {dye }}$ is the absorbance associated to the 3-HF molecules trapped in the film, both evaluated at the excitation wavelength. $A_{d y e}$ is calculated from the ratio between the transmittance spectrum of the film and the transmittance spectrum estimated for the polymeric matrix in which the dye molecules are trapped. Details of the method are provided in the supporting information (Section S3).

$$
\Phi=\eta\left(A_{\text {film }} / A_{\text {dye }}\right)
$$

Time-resolved fluorescence decays were collected using the time correlated singlephoton counting (TCSPC) option of the FluoroLog 3. A NanoLED350 illuminating at 345 $\mathrm{nm}$ with a repetition rate of $1 \mathrm{MHz}$ and a full width at half maximum of $1 \mathrm{~ns}$ was used to excite the sample. The signals were recorded by using an IBH Data Station Hub photon counting module, and data analysis was performed by using the commercially available DAS6 software (HORIBA Jobin-Yvon IBH). The quality of fit was assessed by minimizing the reduced chi squared function $\left(\chi_{R}{ }^{2}\right)$ and by visual inspection of the weighted residuals. 
UV-detection experiments were performed in the spectrofluorometer by regulating the power density of the excitation beam with the aperture of the excitation slits. For these experiments, the samples were irradiated thought a mask to avoid changes in the area of the illuminated zone. A UV light detector (PMA2100 from Solar Light Co., Inc.) was placed in the same position than the samples to measure the irradiance of the excitation beam.

The homogeneity of the deposition process was tested by optical, luminescence and AFM techniques over an area of $4 \mathrm{~cm}^{2}$.

\section{RESULTS AND DISCUSSION}

\subsection{Composition, structure and microstructure of the PAVD thin films}

The 3-HF films grown by PAVD are homogeneous, stable and well adhered to the substrate. All of them are completely insoluble in water and toluene. Furthermore, they are thermally stable (vacuum heating) at least up to $150 \circ \mathrm{C}$, a temperature higher than the one used for the vacuum sublimation of 3-HF molecules. Such characteristics are similar to those observed in other PAVD films. ${ }^{8,10,11,13}$ In these previous studies, it was established that they consist in a cross-linked matrix formed by the binding of the different molecular moieties produced by the plasma fragmentation of the sublimated precursor. In order to confirm these structural characteristics the 3-HF PAVD films have been characterized by XPS, ToF-SIMS and FT-IR spectroscopies.

XPS analysis revealed that changing the deposition parameters has a weak influence on the atomic surface composition of the films within the studied range. This is clearly illustrated by the comparison of the surface composition of samples $3 \mathrm{HF}-1$ ( $74 \% \mathrm{C}$ and $26 \% \mathrm{O}$ ) and $3 \mathrm{HF}-6$ (79\% $\mathrm{C}$ and $21 \% \mathrm{O}$ ) deposited under conditions of maximum and minimum interaction of the sublimated dye molecules with the plasma (see Table 1). At first sight, these compositions are close to that derived from the formula of the dye molecule ( $83 \% \mathrm{C}$ and $17 \% \mathrm{O})$. Note that the surface oxygen enrichment observed in these films is a general phenomenon in plasma polymer that has been also reported for PAVD thin films. ${ }^{9-13}$ This oxygen incorporation is usually attributed to the reaction of surface radicals with air when the films are exposed to the atmosphere after their preparation. ${ }^{46}$

To get information about the chemical structure of the 3-HF PAVD films and confirm the incorporation of entire dye molecules, the ToF-SIMS spectra of selected 3-HF films are presented in Fig. 1a. The reference SIMS spectrum of the 3-HF powder sample is clearly dominated by a peak group centred at $\mathrm{m} / \mathrm{z} \sim 239$ that corresponds to the $\mathrm{MH}^{+}$ molecular ions of 3-HF. On the other hand, SIMS spectra of the PAVD films are characterized by a series of peak groups at $\mathrm{m} / \mathrm{z} \sim 28,43,55,77,91,105,121$ and 165. Some of these peaks were previously detected by mass spectroscopy during glow discharge sublimation of $3-\mathrm{HF} .{ }^{47}$ Most of these peaks correspond to light $(\mathrm{m} / \mathrm{z}<100)$ $\mathrm{CH}(\mathrm{O})$ groups, whereas peaks at $\mathrm{m} / \mathrm{z} \sim 105,121$ and 165 are associated to $\mathrm{C}_{7} \mathrm{H}_{5} \mathrm{O}_{2}$, $\mathrm{C}_{7} \mathrm{H}_{5} \mathrm{O}$ and $\mathrm{C}_{9} \mathrm{H}_{9} \mathrm{O}_{3}$ heavier groups. These latter fragments can be related to the breaking of the central ring of the 3-HF molecule, a mechanism that has been proposed as the most likely for the cleaving of this of compounds. ${ }^{48}$ Precursor fragmentation occurs in some degree in the mass spectrometer, as indicated by the low intense peaks at $\mathrm{m} / \mathrm{z}<$ 238 present in the reference spectrum of the 3-HF powder. Nevertheless, comparison of the PAVD spectra with the reference spectrum indicates that most of the molecular fragments detected at $m / z<238$ in the PAVD samples correspond to the breaking of the precursor molecule during the plasma synthesis. Furthermore, when comparing the SIMS spectra of the samples, it is also apparent the increase in the relative abundance 
of lighter groups at expenses of the heavier groups, when moving from sample 3HF-6 to $3 \mathrm{HF}-1$. Since these spectra were acquired under the same analysis conditions, this trend indicates that an increase in the fragmentation degree of the precursor molecules occurs when deposition parameters change from the conditions yielding 3HF- 6 to those producing $3 \mathrm{HF}-1$ samples. These observations confirm the major role of the interaction between the plasma and the sublimated molecules in the fragmentation process, and are consistent with the formation of a plasma polymeric matrix from the generated moieties. The most remarkable feature in Fig. 1a is that all the spectra present peak groups centred at $\mathrm{m} / \mathrm{z} \sim 239$ and 238 assigned to the $\mathrm{M}^{+}$and $\mathrm{MH}^{+}$molecular ions of 3HF. This finding confirms the retention of the integer precursor structure in the films. Fig. $1 \mathrm{~b}$ shows the evolution of the normalized intensity (see experimental section) of the $\mathrm{m} / \mathrm{z} \sim 239$ peak attributed to the $\mathrm{MH}^{+}$molecular ion of the 3-HF. The trend observed in this figure indicates that the relative abundance of entire dye molecules increases from samples $3 \mathrm{HF}-1$ to $3 \mathrm{HF}-6$ and illustrates the possibility of the technique for the deposition of plasma polymer films with a controlled concentration of functional molecules. 

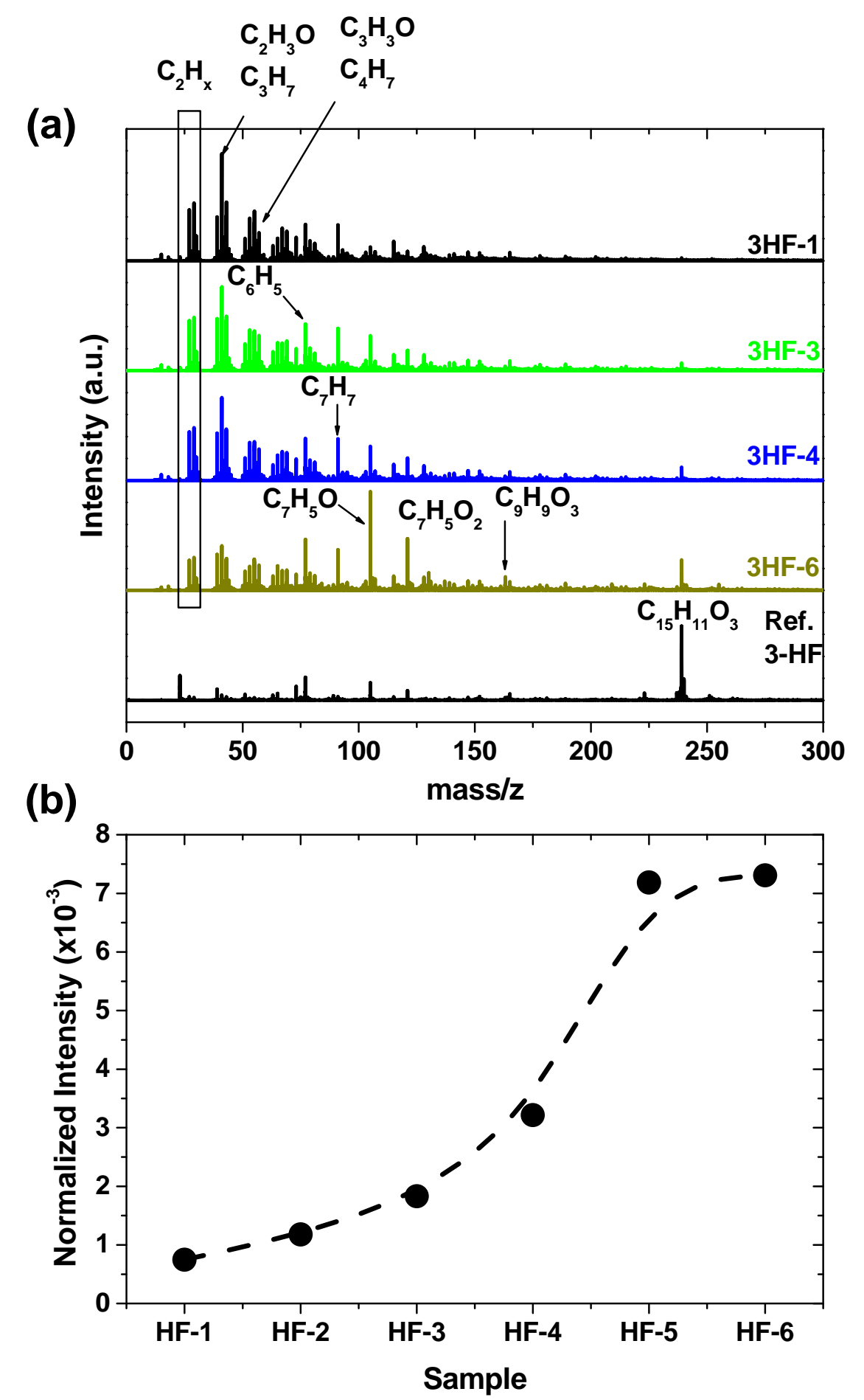

Fig. 1 ToF-SIMS analyses of a set of the 3-HF PAVD films deposited using the conditions in Table 1. (a) ToF-SIMS spectra including a reference spectrum collected for 3-HF powder. (b) Normalized intensity of the peak at $\mathrm{m} / \mathrm{z}=239$ assigned to the $\mathrm{MH}^{+} \mathrm{molecular}$ ions of $3-$ HF.

FT-IR spectra of the 3-HF PAVD films are presented in Fig. 2 in comparison with the spectrum of 3-HF powder in $\mathrm{KBr}$. The latter depicts the characteristics IR features of the 3-hydroxyflavone molecule, ${ }^{49-54}$ which have been attributed as reported in Table S1 provided as supporting information. The IR spectra of the plasma films reproduce most of the 3-HF bands (e.g., peaks at 1609, 1561, 760 and $700 \mathrm{~cm}^{-1}$ ). The features in the PAVD spectra are broad and slightly shifted in position with respect to the molecular spectrum. These structural characteristics are expected for films formed by fragments 
coming from the partial breaking of the dye molecule and by integer 3-HF molecules. Moreover, the film spectra have other additional IR features, not present in the 3-HF spectrum, which are related with the plasma polymeric matrix. Attribution of these new bands can be found in the literature, ${ }^{54-56}$ and in the supporting information (see Tables S2 and S1). Thus, for instance, within the $1600-1800 \mathrm{~cm}^{-1}$ spectral region the peak at $1609 \mathrm{~cm}^{-1}$ matches with the carbonyl stretching modes of the $3 \mathrm{HF}$ molecule, whereas the rest of peaks correspond to carbonyl and alkene stretching vibrations in the new structures formed during the deposition process. Particularly features at $1 / \lambda>1710 \mathrm{~cm}^{-}$ ${ }^{1}$ are attributed to carbonyl stretching mainly in aliphatic structures likely formed as consequence of the breaking of the 3-HF central ring and the cross-linking of the resulting fragments. This interpretation is supported by the presence of IR features from 2800 to $2960 \mathrm{~cm}^{-1}$ assigned to $\mathrm{CH}_{\mathrm{x}}$ stretching modes in saturated aliphatic chains. The development of these new characteristics, as well as, the broadening of the dye features, confirm the plasma polymeric character of the films. ${ }^{15,57,58}$ Furthermore, most of the new bands attributed to saturated groups (e.g., peaks at 2935 and $1750 \mathrm{~cm}^{-1}$ ) increase in intensity in detriment of the 3-HF bands (e.g. vibrations in aromatic structures observed at $1609 \mathrm{~cm}^{-1}$ and between $780-760 \mathrm{~cm}^{-1}$ ) from sample 3HF-6 to $3 \mathrm{HF}-1$. This trend indicates an increase of the dye fragmentation because of the interaction with the plasma species, in agreement with the SIMS results. This behaviour is accompanied by the enhancement of the broadening of the IR features (particularly at $1 / \lambda<1500 \mathrm{~cm}^{-1}$ ) suggesting that the cross-linking and polymerization degree of the matrix varies in this direction. To analyse the evolution of the cross-linking degree of the samples we propose a $\alpha$ parameter defined as the ratio between the area of the 3hydroxyflavone bands between 790 and $680 \mathrm{~cm}^{-1}$ and the area of the $\mathrm{CH}_{\mathrm{x}}$ stretching bands characteristic of the plasma polymer formation in the $3000-2800 \mathrm{~cm}^{-1}$ region. The obtained values are summarized in Table 1 together with other luminescent parameters of the films.

As previously mentioned an evolution in the relative abundance of saturated and unsaturated groups is observed. To analyse this trend Fig. $2 \mathrm{~b}$ shows in a enlarge scale the $\mathrm{CH}_{\mathrm{x}}$ stretching modes of the PAVD films. In this figure it is apparent the increase of the relative intensity of the $\mathrm{CH}_{\mathrm{x}}$ features in saturated groups $\left(\mathrm{CH}_{2}\right.$ and $\mathrm{CH}_{3}$ stretching at 2875 and $2935 \mathrm{~cm}^{-1}$ ) in comparison to those of unsaturated structures ( $\mathrm{CH}$ stretching in olefinic and aromatic groups at 3035 and $3065 \mathrm{~cm}^{-1}$ respectively) from sample $3 \mathrm{HF}-6$ to $3 \mathrm{HF}-1$. In summary, ToF-SIMS and FT-IR analyses indicates that the PAVD process renders plasma polymeric films with different structural characteristics (e.g. fragmentation and cross-linking degree, abundance of saturated groups, retention of precursors structure among others) depending on the deposition parameters, but preserving a substantial amount of non-fragmented precursor molecules. These different characteristics modify the film optical properties and luminescent behaviour of the trapped dye molecules as it is discussed below. 

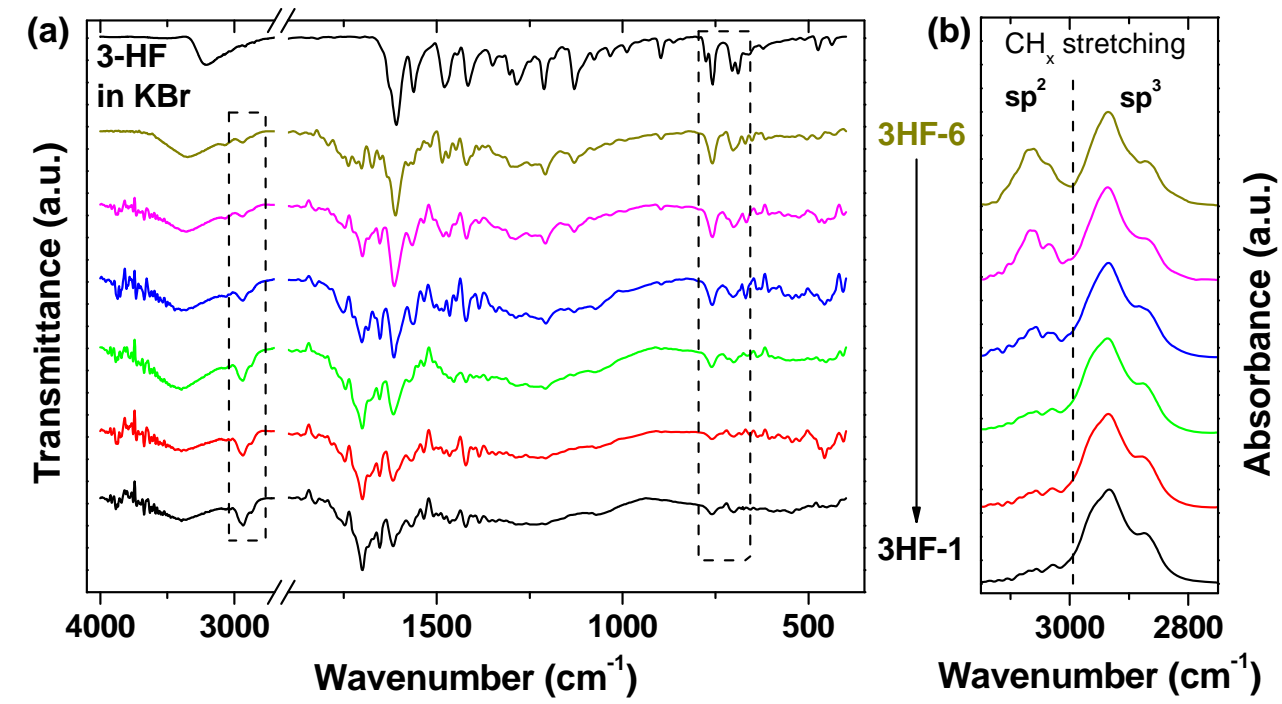

Fig. 2 (a) FT-IR spectra of the 3-HF PAVD films. The spectrum of 3-HF powder in $\mathrm{KBr}$ pellet is included for comparison. (b) Detail of the $\mathrm{CH}_{\mathrm{x}}$ vibrations (absorbance scale) at the $2750-3150 \mathrm{~cm}^{-1}$ spectral region. The arrow indicates the evolution from spectra $3 \mathrm{HF}-6$ to $3 \mathrm{HF}-1$.

Table 1 Growth parameters and structural and luminescent characteristics of the 3-HF PAVD films

\begin{tabular}{|c|c|c|c|c|c|}
\hline \multirow{2}{*}{ Sample } & \multicolumn{2}{|c|}{ Growth Parameters } & \multirow{2}{*}{$\begin{array}{c}\text { Cross-linking } \\
\text { parameter } \\
\alpha^{\mathrm{c}}\end{array}$} & \multicolumn{2}{|c|}{$\begin{array}{c}\text { Luminescent } \\
\text { properties }\end{array}$} \\
\hline & $\begin{array}{c}\mathrm{z}^{\mathrm{a}} \\
(\mathrm{cm})\end{array}$ & $\begin{array}{c}\mathrm{r}^{\mathrm{b}} \\
\left(\mathrm{nm} \mathrm{min} \min ^{-1}\right)\end{array}$ & & $\begin{array}{l}\eta^{d} \\
(\%)\end{array}$ & $\begin{array}{c}\Phi^{\mathrm{e}} \\
(\%)\end{array}$ \\
\hline $3 \mathrm{HF}-1$ & 1.0 & 0.48 & 5.1 & 0.2 & 0.7 \\
\hline $3 \mathrm{HF}-2$ & 5.2 & 0.48 & 4.0 & 0.2 & 0.7 \\
\hline $3 \mathrm{HF}-3$ & $1.0^{*}$ & 0.36 & 3.2 & 0.2 & 0.6 \\
\hline $3 \mathrm{HF}-4$ & $5.2 *$ & 0.36 & 1.1 & 0.7 & 1.2 \\
\hline $3 \mathrm{HF}-5$ & $5.2 *$ & 0.48 & 0.31 & 1.9 & 2.7 \\
\hline $3 \mathrm{HF}-6$ & $5.2 *$ & 0.6 & 0.29 & 2.5 & 3.4 \\
\hline
\end{tabular}

*Samples deposited using the front configuration.

${ }^{\mathrm{a}}$ Distance between the deposition and plasma zones.

${ }^{\mathrm{b}}$ Corrected deposition rate.

${ }^{\mathrm{c}}$ Cross-linking parameter obtained from FT-IR spectra in Fig. 2a as indicated in the text.

${ }^{\mathrm{d}}$ External radiative quantum efficiency of the green emission of the films. ${ }^{44}$

${ }^{\mathrm{e}} \mathrm{Quantum}$ yield of the green emission 3-HF molecules embedded in the films.

Typical organic layers prepared by direct dye vacuum sublimation in absence of plasma are formed by non-homogenous and weakly adhered microcrystallites, whose morphology and size change with the time. ${ }^{9,10,47}$ These crystallites disperse the light strongly. By contrast, the thin films prepared by PAVD are characterized by a continuous and regular surface. As a representative example, the AFM images of a thin $(\sim 90 \mathrm{~nm})$ and a thick ( $305 \mathrm{~nm})$ PAVD film deposited using the conditions of sample 3HF-6 are presented in Fig. 3. They clearly evidence that the 3-HF PAVD films have a very homogeneous, crack free and smooth surface with a root mean squared (RMS) roughness of $\sim 0.3 \pm 0.05 \mathrm{~nm}$. It is worth of note that the RMS roughness does not increase with the thickness in the studied range, from very thin to relatively thick films. The presented RMS values stand for images of $0.5 \mu \mathrm{m} \times 0.5 \mu \mathrm{m}, 1 \mu \mathrm{m} \times 1 \mu \mathrm{m}, 1.5 \mu \mathrm{m} \times$ $1.5 \mu \mathrm{m}$ and $2.5 \mu \mathrm{m} \times 2.5 \mu \mathrm{m}$, meaning that the saturation regime for the RMS has been reached at the lowest measured scale. Statistical analysis has been performed from at least 5 images obtained in different positions of the samples. Similar and reproducible topographic values were measured in different regions of the $1 \times 1 \mathrm{~cm}^{2}$ samples and in samples located in different positions of the $2 \times 2 \mathrm{~cm}^{2}$ sample holder in more than 10 deposition experiments. The homogeneity and flatness of the plasma film is a very important property for the integration of such films as active layer in optical devices. 

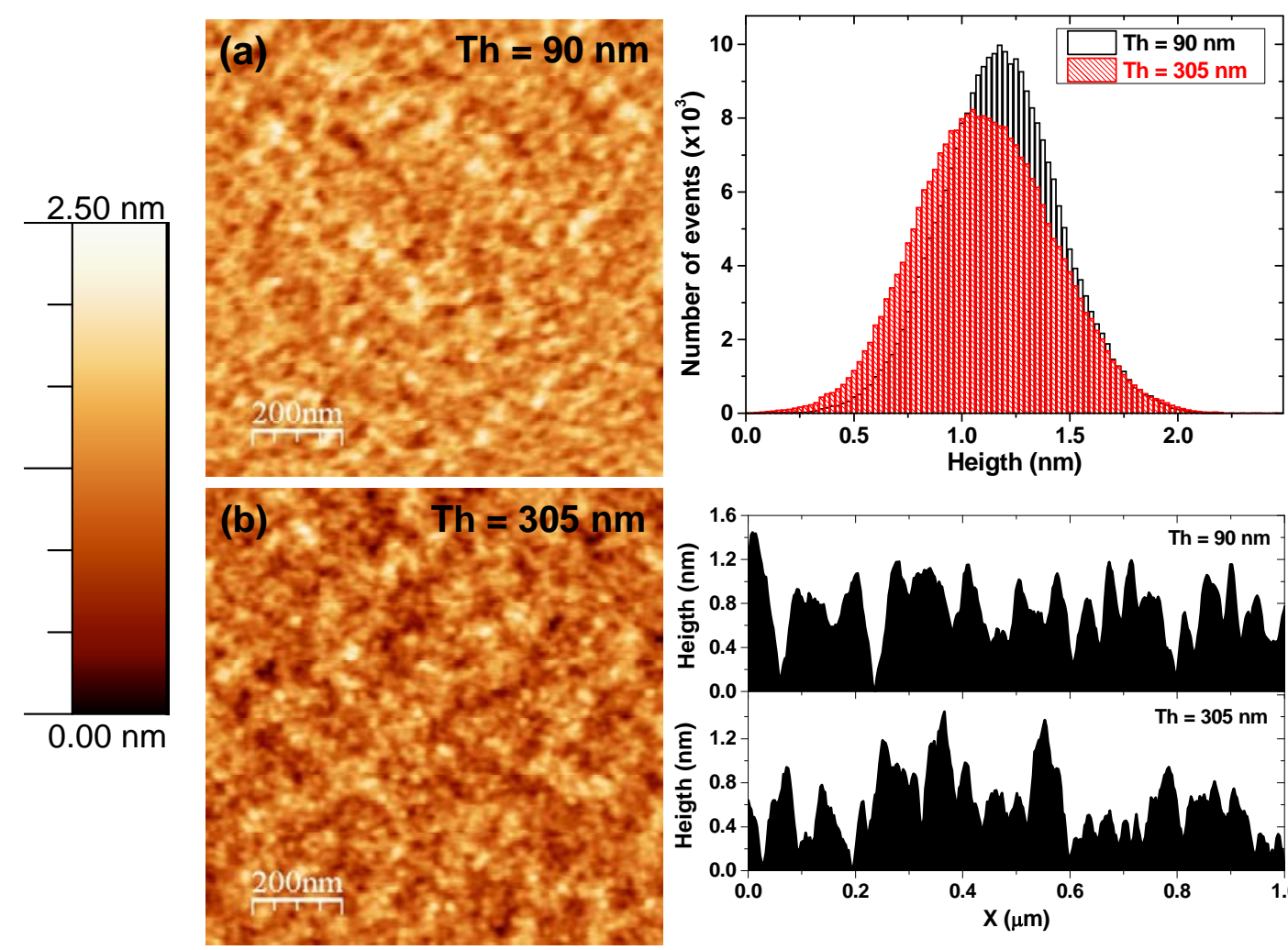

(c)

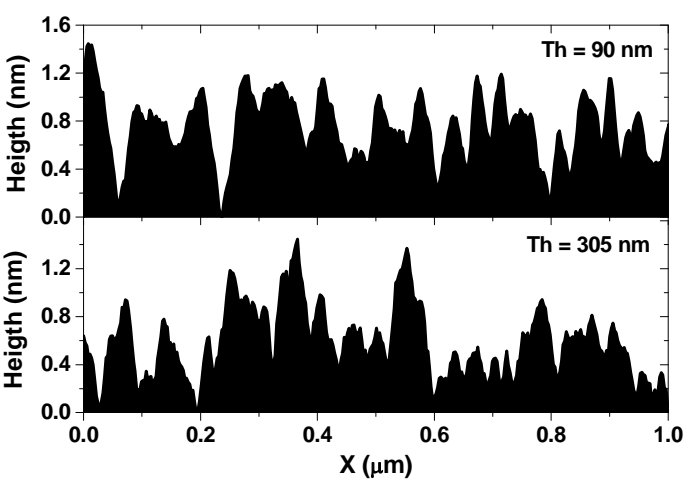

(d)

Fig. 3 Surface topography of two 3HF-6 PAVD films (cf. Table 1) with different thicknesses as indicated in the figure. (a, b) AFM tapping mode images $\left(1 \times 1 \mu \mathrm{m}^{2}\right)$. (c) Height distribution and (d) line profiles of both films.

\subsection{Optical properties of 3-HF PAVD thin films}

The absorbance spectrum of 3-HF in different liquid media ${ }^{30,32,33}$ and in dye doped films prepared by sol gel $\left.\right|^{35,51}$ is characterized by a wide absorption in the near UV region $(400-200 \mathrm{~nm})$ in which it is possible to recognize two well defined absorption bands. These are a main peak at $\sim 340 \mathrm{~nm}$ with a low energy shoulder, both assigned to $S_{0} \rightarrow S_{1}$ electronic transitions and a less intense maximum at $\sim 310 \mathrm{~nm}$, attributed to $S_{0} \rightarrow S_{2}$ transitions. ${ }^{59}$ The UV-Vis spectra of a set of 3-HF thin films ( $\left.175 \mathrm{~nm}\right)$ are represented in Fig. 4a. The transmittance spectrum of sample 3HF-1 is characterized by a continuous absorption at wavelengths below $390 \mathrm{~nm}$ that is attributed to $\pi \rightarrow \pi^{*}$ transitions involving the unsaturated bonds of a cross-linked polymeric matrix. This absorption is a common feature in organic plasma polymers in general. ${ }^{8,9,60,61}$ Superimposed on this absorption background, in the spectrum of sample $3 \mathrm{HF}-2$ it is possible to recognize two low intense absorption bands at $\sim 310$ and $345 \mathrm{~nm}$ that confirm the presence of active $3-\mathrm{HF}$ molecules in the film. The intensity of these dye bands is controlled by the deposition parameters and it progressively develops from sample $3 \mathrm{HF}-1$ to $3 \mathrm{HF}-6$, in agreement with the increase in the concentration of $3-\mathrm{HF}$ molecules previously observed by ToF-SIMS. Thus, under conditions of minimum interaction the absorbance at $350 \mathrm{~nm}$ reach values of $c a$. 0.6 (i.e. absorptance $>70 \%$ after substrate correction) for a thin films of $\sim 175 \mathrm{~nm}$ of thickness. Furthermore the absorption of UV light can be easily improved by increasing the thickness of the sample as illustrated in Fig. $4 \mathrm{~b}$ corresponding to a 3HF-6 thin film of $\sim 305 \mathrm{~nm}$ of thickness. In this film, the absorption in the UV region is nearly complete, whereas at wavelengths higher than $390 \mathrm{~nm}$ the film is transparent showing only the typical interference fringes due to the refractive index contrast between the substrate and the film. Saturation of the UV absorption, transparency in the visible region and sharpness of the absorption edge are requirements for the fabrication of UV-filters, an application where, according to 
spectra in Fig. 4, this kind of films can compete in performance with thin-film materials prepared by other methods. ${ }^{62}$

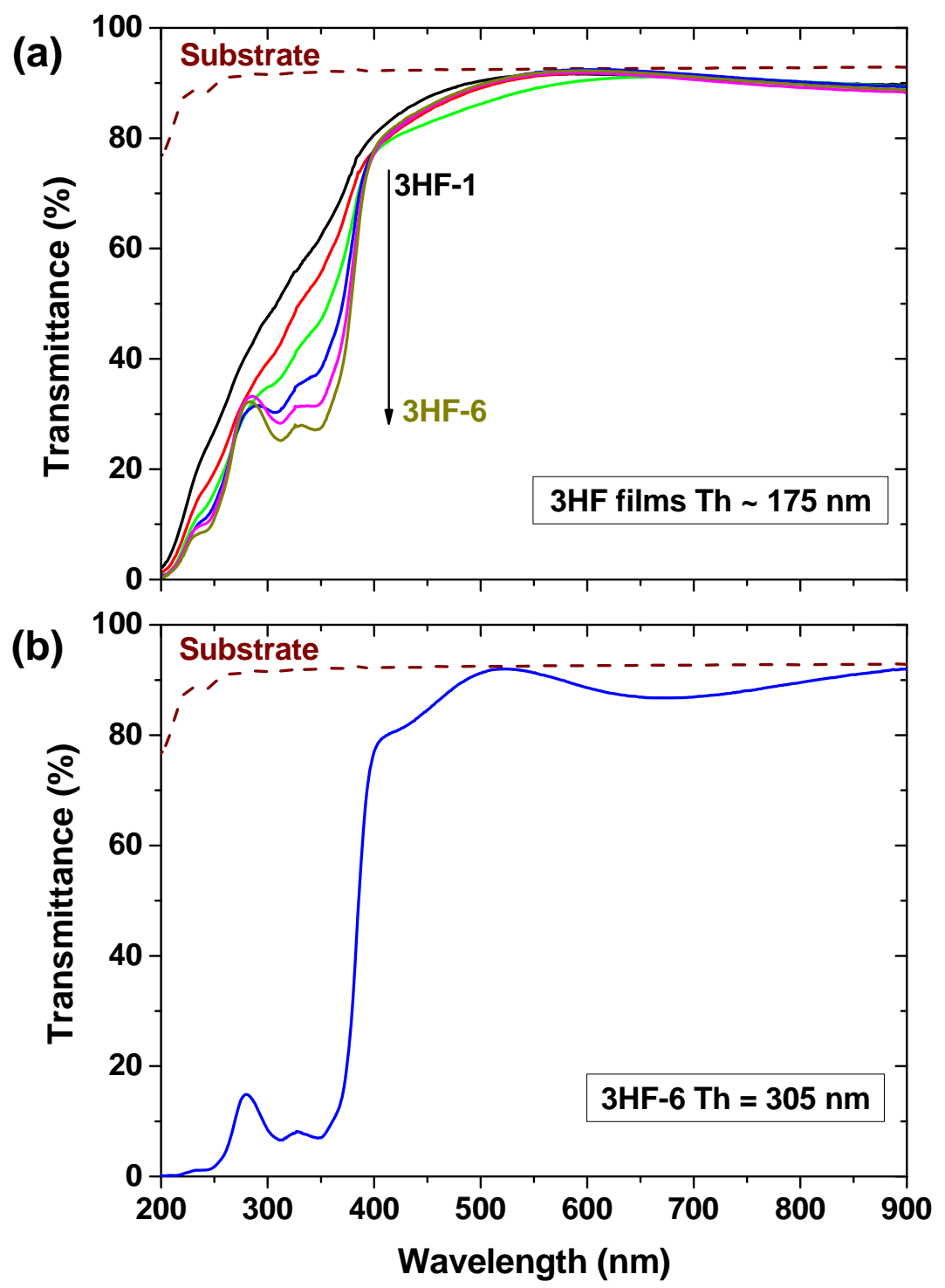

Fig. 4 (a) UV-Vis spectra of 3-HF PAVD thin films ( 175 nm). (b) UV-Vis spectrum of a $3 \mathrm{HF}-6$ film with a thickness of $305 \mathrm{~nm}$. The transmittance spectrum of a fused silica substrate is included in each figure.

In order to further assess the optical quality of the films, their extinction coefficient (k) and refractive index $(\mathbf{n})$ have been determined by spectroscopic ellipsometry (Fig. 5 ). In agreement with the transmittance spectra, the $\mathbf{k}$ vs. $\lambda$ curves present a continuous UV absorption background and, superimposed on it, the typical absorption characteristic of the 3-HF molecules. The intensity of these absorption features increases from sample $3 \mathrm{HF}-1$ to $3 \mathrm{HF}-6$. For wavelengths higher than $390 \mathrm{~nm}$, the extinction coefficient sharply decreases to reach values lower than $2 \times 10^{-3}$ at $550 \mathrm{~nm}$, the sensitivity limit of the ellipsometer. Transparency in the visible and NIR regions was an important requirement for the integration of these films in resonant photonic structures. ${ }^{6}$ Such practical applications in the photonic field also require an accurate control of the refractive index of the films. Among other strategies, incorporation of unsaturated bonds and particularly aromatic rings is one of the approaches used to increase the refractive index of polymeric materials. ${ }^{63-65}$ This is so thanks to the high polarizability of these structures. In our case, FT-IR analysis shows that the deposition approach enables regulating the retention of such moieties over a significant range (note the trend 
presented in Fig. $2 \mathrm{~b}$, and see the evolution of the $\alpha$ parameter in Table 1). Thus, the refractive index of the films diminishes from samples 3HF-6 to 3HF-1 (e.g., $\mathbf{n}$ values of 1.67 and 1.58 can be measured at $632.8 \mathrm{~nm}$ for samples $3 \mathrm{HF}-6$ and $3 \mathrm{HF}-1$, respectively). It is worth of note that the reported refractive index values cover a rather wide range reaching relatively high values when comparing to other plasma polymeric films or even to conventional optical polymers. ${ }^{8,26,63,66-68}$
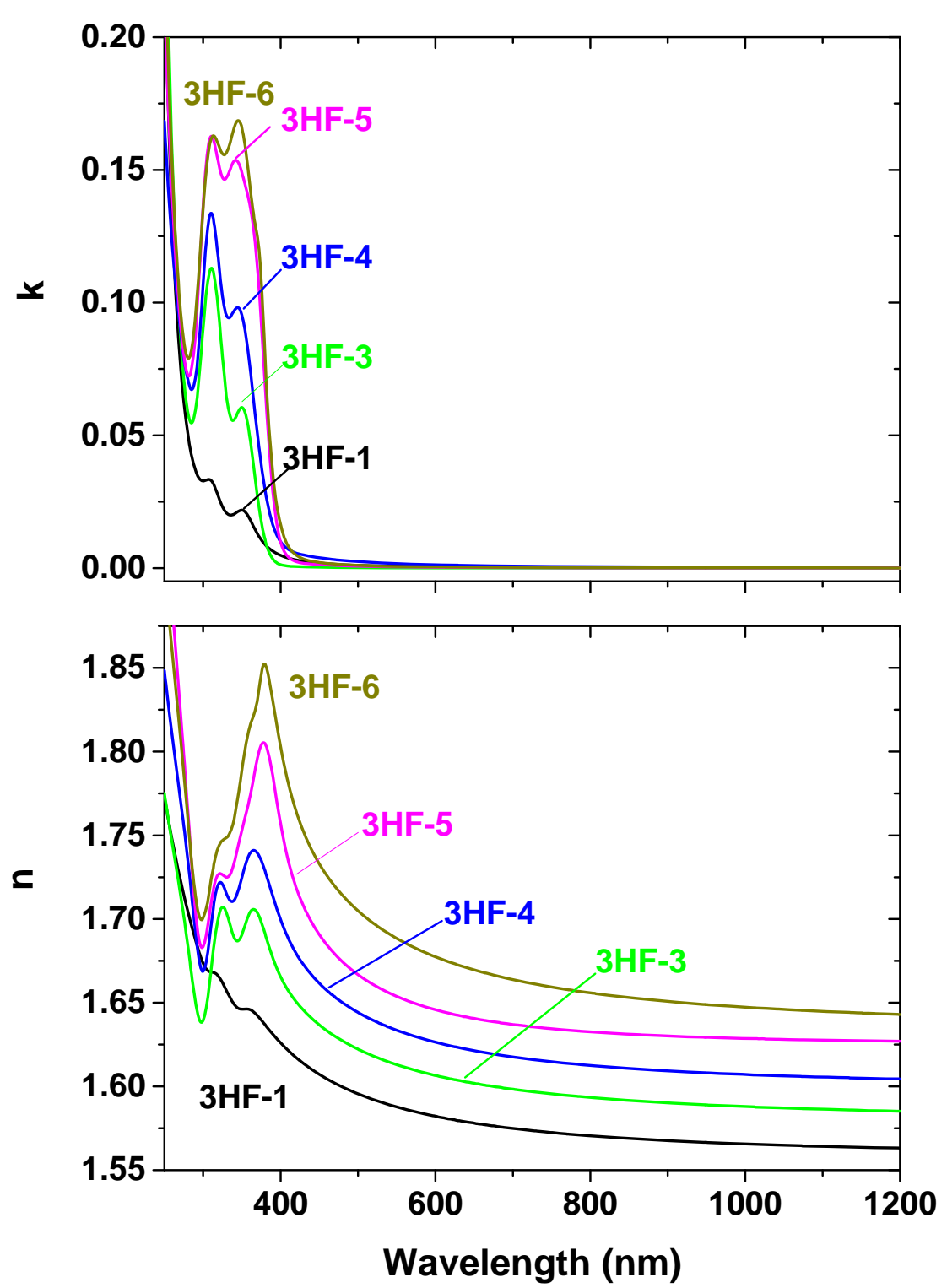

Fig. 5 Extinction coefficient $\mathbf{k}$ and refractive index $\mathbf{n}$ of 3-HF PAVD thin films ( $\sim 175 \mathrm{~nm}$ ).

\subsection{Steady state and time resolved fluorescence}

A relevant characteristic of the 3-HF molecule is that it exhibits a dual blue and green emission when it is excited with UV light. The blue emission (around $425 \mathrm{~nm}$ ) is due to the normal form of this molecule $(\mathrm{N})$, whereas the green emission (around $530 \mathrm{~nm}$ ) is assigned to its tautomeric form $(T) .^{30-32,35}$ The fluorescence emission spectra of the 3-HF PAVD films are reported in Fig. 6a. For all the analysed samples the most intense feature, at $\sim 535 \mathrm{~nm}$, corresponds to the $\mathrm{T}$ band of the $3-\mathrm{HF}$ molecules, since the corresponding excitation spectra are similar to those reported for this compound in solution (see excitation spectrum of the sample 3HF-6 provided as example in Fig. S3). On the other hand the $\mathrm{N}$ emission $(400-450 \mathrm{~nm})$ is very weak or even negligible for 
samples $3 \mathrm{HF}-4$ to $3 \mathrm{HF}-6$. This result indicates that the proton transfer required for the $\mathrm{N}$ to $\mathrm{T}$ conversion of 3-HF is a very highly efficient process in the studied PADV films and, therefore, suggests a low hydrogen donor capability of the matrix where the molecules are embedded. A similar behaviour has been reported for non-polar and aprotic solutions of this dye. ${ }^{31-33}$ Comparing the series of spectra in Fig. 6a, it is also apparent that the relative intensity of the $\mathrm{N}$ band decreases from sample $3 \mathrm{HF}-1$ to $3 \mathrm{HF}-6$. This tendency must be taken as an indication of an increasing efficiency of the ESIPT process in that direction and related to the differences observed by FT-IR in the structural characteristics of the plasma polymeric films.

The emission intensity at $530 \mathrm{~nm}$ as a function of the absorptance at the excitation wavelength $\left(\lambda_{\mathrm{ex}}=350 \mathrm{~nm}\right)$, is plotted in Fig. $6 \mathrm{~b}$. This discloses an increase in the fluorescence efficiency starting in sample $3 \mathrm{HF}-3$ up to sample 3HF-6. To quantitatively asses this emission enhancement, we have calculated the quantum yield ( $\Phi$ in Table 1 ) of the green emission from the dye molecules embedded in the film. The luminescence quantum yield seems to be constant and have a low value for films $3 \mathrm{HF}-1$ to $3 \mathrm{HF}-3$, whereas it increases from $0.6 \%$ to $3.4 \%$ from samples HF-3 to HF-6. It is interesting to comment here that despite this low value, the green fluorescence emission from the films can be directly observed with the naked eyes even in ambient light conditions (see Fig. 7). This is due to the very high dye concentration in the films (strong UV-absorption) and also to the absence of luminescence autoabsorption as it is discussed below. As it is indicated in the supporting information (see Section S7 about the different processes involved in the green emission of the 3-HF molecules), the quantum yield of the green emission of the 3-hydroxyflavone is proportional: i) to the efficiency of the ESIPT process and ii) to the efficiency of the radiative deactivation process from the $T$ excited state. Thus, it is clear that the evolution of $\Phi$ in Table 1 is partially related to the increase of the ESIPT efficiency inferred from the spectra in Fig. 6b. Nevertheless, differences in the cross-linking degree of the polymeric matrix and/or changes in the type of polymeric structure around the molecules (cf. IR analysis in Section 3.1) are factors that, besides disturbing the ESIPT, might modify the efficiency of the radiative deactivation process from the $T^{*}$ emitting state. Thus, the observed variation in the value of $\Phi$ could be also accounted by this latter effect. 

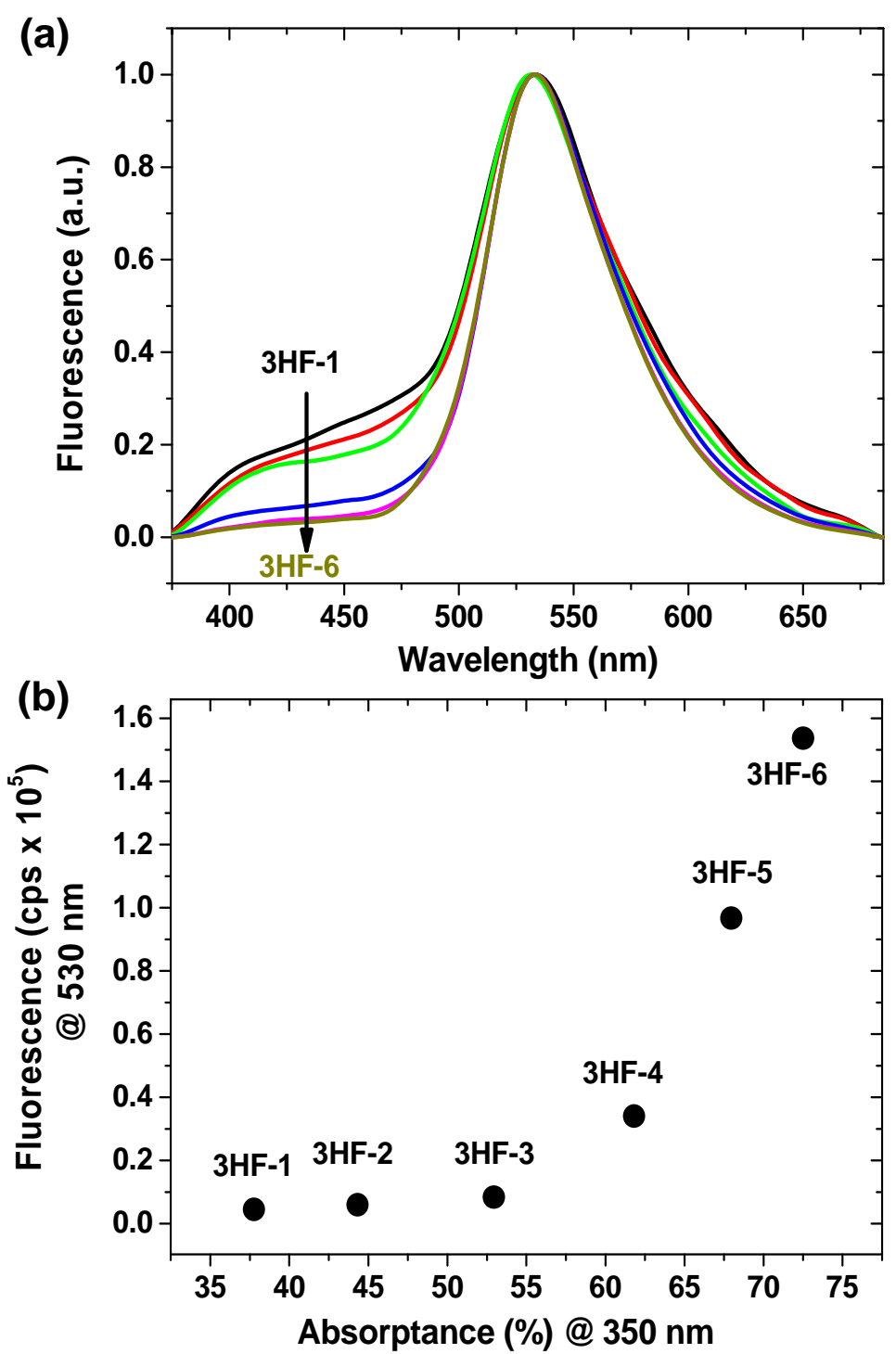

(c)

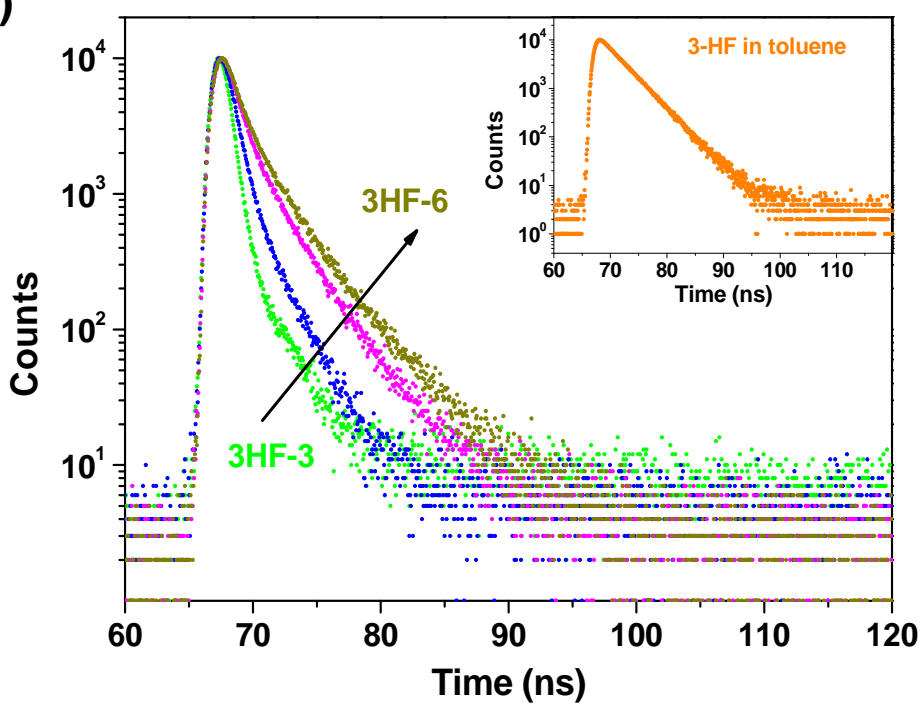

Fig. 6 Luminescent properties of the 3-HF PAVD thin films $\left(\lambda_{\mathrm{ex}}=350 \mathrm{~nm}\right)$. (a) Height normalized emission spectra. The arrow indicates the evolution from 3HF-3 to 3HF-6 samples. (b) Fluorescence intensity at $530 \mathrm{~nm}$ as a function of the absorptance at the excitation wavelength. (c) Fluorescence decay curve at $530 \mathrm{~nm}$. The inset corresponds to the fluorescence decay curve of 3-HF in toluene (10-6 $\mathrm{M}$ ). 
A way to ascertain the influence of the surrounding medium into the radiative deactivation of the $T^{*}$ state is to measure the lifetime of the T emission. With this aim, the fluorescence decay curves of the emission at $530 \mathrm{~nm}$ have been acquired by nanosecond time correlated single photon counting technique (Fig. 6c). Taking into account that the emissive $T^{*}$ state is not directly populated by photo-excitation but by a post-ESIPT process (see Fig. S5), the fluorescence decay curves should consist in a growing component (formation of emissive $T^{*}$ state) and a decay component (deactivation of $\mathrm{T}^{*}$ state). However, neither of the recorded fluorescence decay curves of 3-HF films have a growing component, suggesting the very-fast dynamic of the ESIPT process, faster (sub-ns) that the time resolution of the experimental set-up. Indeed the kinetics of the ESIPT process of the $3-\mathrm{HF}$ molecule is in the ps and fs time-scale. ${ }^{69}$ For the sake of comparison it is also analysed the life time of the emission from 3-HF in toluene (inset in Fig. 6c), a system where the quantum yield of the dye molecule reaches its highest value. ${ }^{70}$ This fluorescence decay curve can be described by a monoexponential function with a lifetime of 3.5 ns. Meanwhile emission decay curves of samples 3HF-4 to 3HF-6 have to be adjusted to at least a tri-exponential function, with a $\chi_{R}{ }^{2}$ value lower than 1.2 and Durbin-Watson statistical parameter higher than 1.7. For the emission of sample 3HF-6 this fitting model renders lifetimes of 0.5 ns (26\%), $1.9 \mathrm{~ns}(50 \%)$ and $4.3 \mathrm{~ns}(24 \%)$. Similar lifetimes are also obtained for samples $3 \mathrm{HF}-5$ and 3HF-4 but with different relative contributions (cf. Table 2). This triple fitting scheme should be associated to dye molecules trapped in different local environments where the fluorescence would be differently quenched. ${ }^{69}$ Thus, the degree of local heterogeneity must be higher for samples $3 \mathrm{HF}-1$ to $3 \mathrm{HF}-3$ with almost similar decay curves that cannot be fitted by using a tri-exponential model. Assuming that the faster components arise from the most effectively quenched 3-HF molecules, the calculated decay models and the area under the decays curves indicate that the average efficiency of the T emission increases from samples 3HF-3 to 3HF-6. All these results suggest that the reduction of the quenching processes of the tautomeric species is one of the factors responsible of the quantum yield enhancement observed when comparing the 3-HF containing films ( $c f$. Table 1 ).

In summary, the previous fluorescence analyses (steady state and time resolved) indicate that the $\Phi$ of the embedded $3-\mathrm{HF}$ molecules and therefore the fluorescence efficiency of the samples can be improved by properly tailoring the chemical environment of the dye molecules. The efficiency of the ESIPT process populating the $T^{*}$ level (Fig. 6a) and of the radiative deactivation from this excited state (Fig. 6c) are the main factors involved in this enhancement. The first one is associated to the capability of the dye to establish hydrogen bonds with the surrounding media. ${ }^{30-32,35}$ Whereas the observed increase in the efficiency of the radiative desexcitation might be tentatively related to the structural characteristics of the different matrix (cf. Figs. 1 and 2 and table 1) where the molecules are embedded. This result paves the way to increase the emission intensity by controlling the composition and structural characteristics of the plasma polymeric matrix. With this purpose, a new set of depositions by making use of an additional precursor is at present under study. 


\begin{tabular}{|c|c|c|c|c|c|}
\hline Sample & $\tau_{1}\left(f_{1}\right)$ & $\tau_{2}\left(\mathrm{f}_{2}\right)$ & $\tau_{3}\left(\mathrm{f}_{3}\right)$ & $<\tau_{\mathrm{A}}>(\mathrm{ns})$ & $\chi_{R}^{2}$ \\
\hline $3-\mathrm{HF}$ in toluene & $3.5 \mathrm{~ns}$ & & & 3.5 & 0.97 \\
\hline $3 \mathrm{HF}-4$ & $0.6 \mathrm{~ns}(64 \%)$ & $1.4 \mathrm{~ns}(31 \%)$ & $3.7 \mathrm{~ns}(5 \%)$ & 1.0 & 1.16 \\
\hline $3 \mathrm{HF}-5$ & $0.6 \mathrm{~ns}(38 \%)$ & $1.9 \mathrm{~ns}(49 \%)$ & $4.3 \mathrm{~ns}(13 \%)$ & 1.7 & 1.01 \\
\hline $3 \mathrm{HF}-6$ & $0.5 \mathrm{~ns}(26 \%)$ & $1.9 \mathrm{~ns}(50 \%)$ & $4.3 \mathrm{~ns}(24 \%)$ & 2.1 & 1.19 \\
\hline
\end{tabular}

Typically, fluorescent organic dyes are characterized by rather short Stokes shifts leading to a substantial overlap between the emission and absorption bands and the reabsorption of part of the emitted light. This is an undesirable effect when trying to maximize the emission output of the samples. The T emission in the 3-HF PAVD films is characterized by a large Stokes shift $(\Delta \lambda \sim 180 \mathrm{~nm})$ that precludes a significant overlap between the emission and absorption bands and allows to enhance the light emission by just increasing the film thickness. This concept is proved in Fig. 7a where the emission intensity at $\lambda_{\mathrm{em}}=530 \mathrm{~nm}$ recorded for a set of $3 \mathrm{HF}-6$ films of different thicknesses (transmission spectra of the films herein analysed are included in Fig. S4) is plotted as a function of the absorptance of the films at the excitation wavelength. This figure clearly shows that the emission intensity gradually increases with the absorptance of the films even for the sample with an absorptance of $93 \%$ where no saturation is yet observed (i.e. the 305-nm-thick film in Fig 7a). The rather linear increase of the fluorescence intensity with the absorptance seems to indicate that the films thickness has no influence on the fluorescence efficiency of the film within the studied rage. Moreover the lack of light reabsorption supports the use of this material as active medium in a large variety of applications, for instance: in resonant cavities, lateral propagating photonic crystals, luminescent waveguides and other kind of photonic devices where the emitted light is confined or propagates through the active films; $;^{2,71}$ as well as wavelength shifters or UV photodetectors. ${ }^{37,38}$ The performance of the films for these two latter applications is illustrated with the photographs in Fig. 7 showing the green light emitted by a film when illuminated with UV lamp, both in a dark or in an illuminated room. In both cases the green emission of the films can be clearly appreciated by visual inspection. This figure also illustrates the procedure of producing luminescent motifs by depositing the film through a shadow mask. The dimension of these patterns can be reduced at the micrometric scale depending on the mask. ${ }^{6}$ Besides, the films can be also processed by plasma etching treatments to produce a different kind of patterns. ${ }^{9,10}$ Both approaches allow to selective coat specific areas of photonic and optoelectronic devices. ${ }^{6}$ Pictures in Fig. 7 also show examples of deposition over polymeric and flexible substrates (e.g. polycarbonate) with a coated area in the range of a standard Si wafer dimension (deposition on substrates of $\sim 50$ $\mathrm{cm}^{2}$ ). Note this latter deposit present some inhomogeneities between the sample centre and the edges. This is mainly due to the use of a single sublimation source in our experimental set-up. However, preliminary results indicate this technical issue can be easily solved by scaling up the deposition system using a larger plasma reactor and a cluster sublimation source to increase the coated area. In any case it is interesting to stress that the deposition process can be directly applied to the fabrication of active component in photonic structures and the compatibility of the process with industrial scale fabrication. ${ }^{6,72}$ 


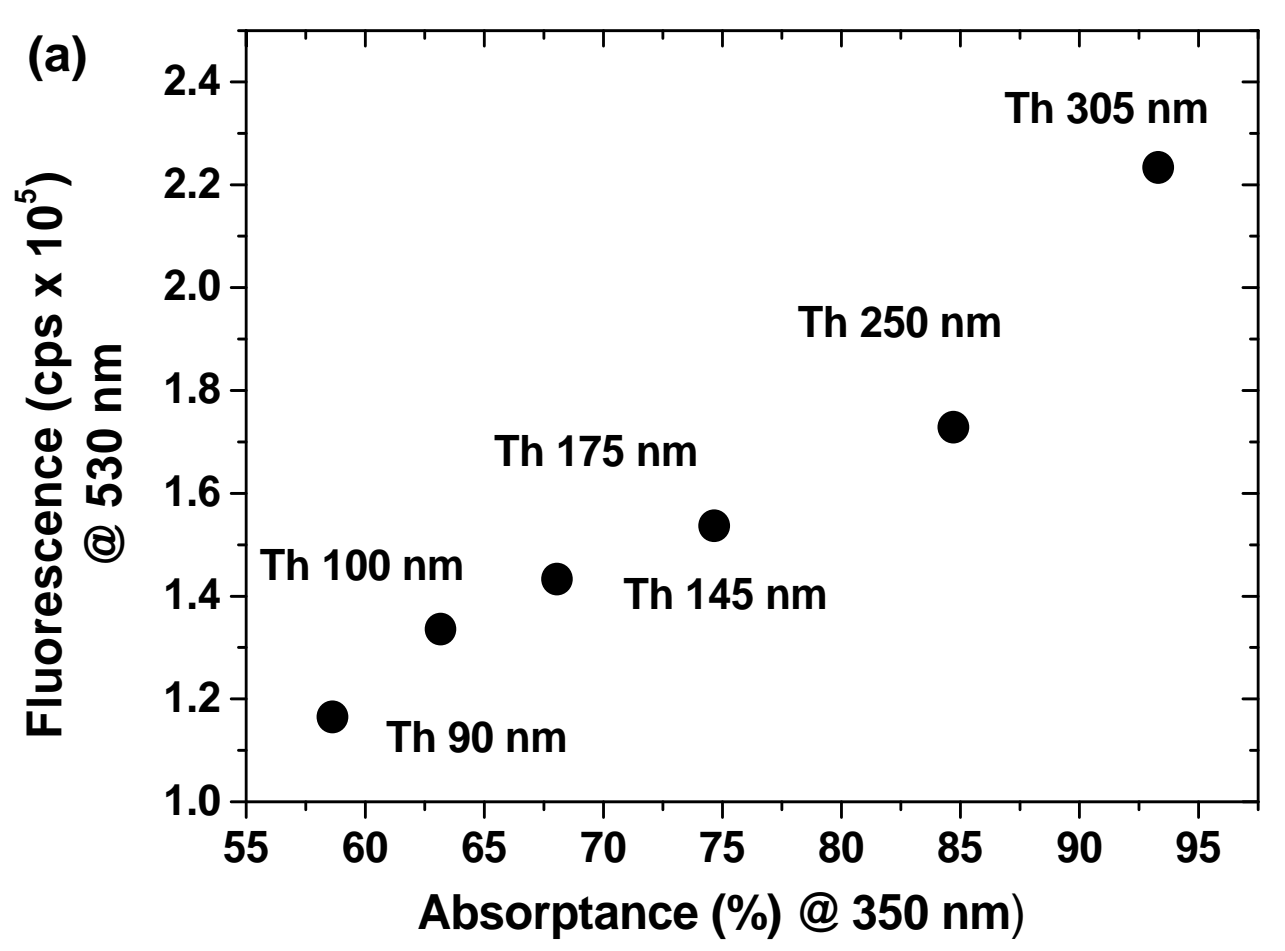

(b)

(c)
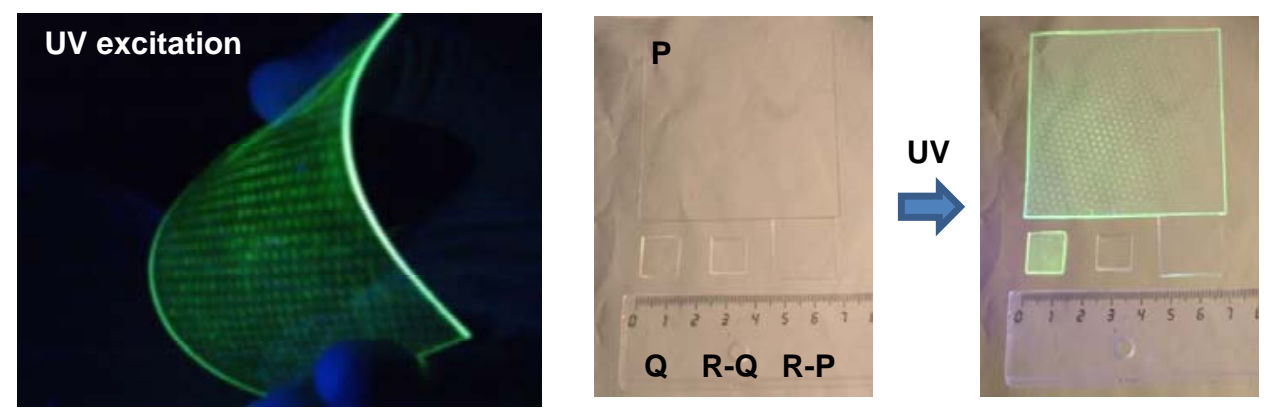

Fig. 7 (a) Fluorescence intensity at $530 \mathrm{~nm}$ as a function of the absorptance at the excitation wavelength $\left(\lambda_{\text {ex }}=350 \mathrm{~nm}\right)$ for a set of $3 \mathrm{HF}-6$ films with different thicknesses as indicated in the figure. (b) Green emission ( $\lambda_{\text {ex }}=365 \mathrm{~nm}$ ) of a sample grown on polycarbonate piece of $\sim 50 \mathrm{~cm}^{2}$. For the deposition a metallic grid was interposed between the substrate and the sublimation cell in order to obtain a periodic luminescent pattern. (c) Photographs of luminescent3-HF films growth on polycarbonate (P) and on a fused silica piece (Q). The images were recorded in a normally illuminated room without (left) and with (right) UV excitation. Reference fused silica (R-Q) and polycarbonate substrates (R-P) are included for the sake of comparison.

\subsection{Response towards UV illumination}

The large Stokes shift $(\Delta \lambda \sim 180 \mathrm{~nm})$ of the green emission of the samples illuminated in the UV region $(\lambda \sim 350 \mathrm{~nm})$ and the lack of fluorescence under visible irradiation make these films good candidates as wavelength shifters and UV photodetectors. An important limitation when intending to employ dye molecules for these applications is that they may degrade after a prolonged exposure to UV light. A limited photodegradation has been in fact observed in our films. However, during prolonged irradiation experiments, we found that after $2 \mathrm{~h}$ of $\mathrm{UV}$ irradiation of $156 \mu \mathrm{W} \mathrm{\textrm {cm } ^ { - 2 }}$, the luminescence of the 3-HF films becomes stable after an initial decrease of $70 \%$ with respect to the original value. The stable response of the $530 \mathrm{~nm}$ emission, for one of these pre-illuminated films, and the respective fluorescence spectra is reported in Fig. 8 for values of irradiance ranging from 1 to $156 \mu \mathrm{W} \mathrm{cm}$. Stability of the emission at 530 $\mathrm{nm}$ during prolonged UV irradiation is presented in top of the figure. The rather good 
photostability of the samples is related to the ESIPT process that the 3-HF molecules undergoes after UV excitation. This is a fast energy dissipation mechanism (typical in the ps-ns range) that decrease the probability of chemical reactions in the UV excited state. Thanks to this quality different kind of ESIPT dyes have been used for the development of UV protective coatings. ${ }^{62}$ In case of 3-HF containing materials it have been reported that the dye photostability depends on the characteristics of the surrounding media. ${ }^{73}$ The fitting of the luminescent decay curves demonstrates that the dye is trapped in different microenvironments that affect their luminescent properties (i.e. the presence of three or more decay terms in Fig. 6c). Hence, the emission decrease observed during the pre-illumination treatment might be related to dye molecules in different local environments where the dye is more effectively photodegradated (data not shown). Fig. 8 also shows that the sensitivity limit of the film to detect low intense UV light is lower than $10 \mu \mathrm{W} \mathrm{cm}$.

A requirement for UV sensing applications is that the intensity of the emitted green light varies linearly with the power density of the excitation beam. A linear response is in fact proved (Fig. 8e) when plotting the emission intensities as function of the illumination densities. This linear behaviour sustains the use of these films as UV detectors and/or green emitters. The extremely high sensitivity of the films towards UV detection is illustrated by the pictures inserted in the Fig. $8 \mathrm{~b}$ showing the green emission from the regions illuminated by an optical fibre bundle. Moreover, due to the high intensity of the green emission, the PAVD films could also be used as visual tags for solar UV detection. The feasibility of this application is proved in Fig. $8 \mathrm{~d}$. In this case the image corresponds to the fluorescent emission, recorded by an inexpensive webcam, when the film is illuminated with solar UV light through a $340 \mathrm{~nm}$ band pass filter. It is important to stress that due to the high intensity of the fluorescence of the film, it was necessary to interpose an additional $2.5 \%$ neutral filter between the excitation and the sample in order to avoid saturation effects in the acquisition of this image. A similar approach can be used to detect indirect sunlight emission (data not shown). 
(a)

(d)

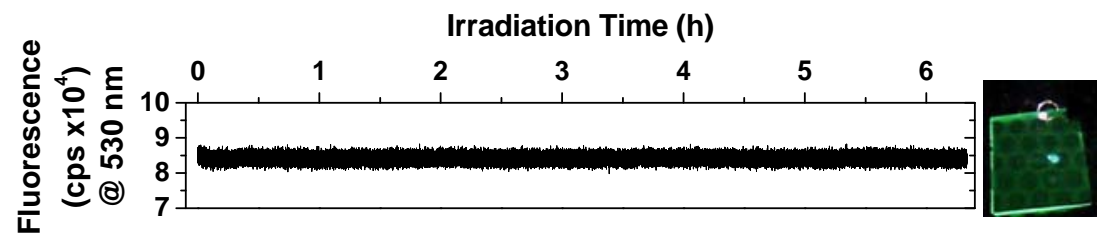

(b)

(c)

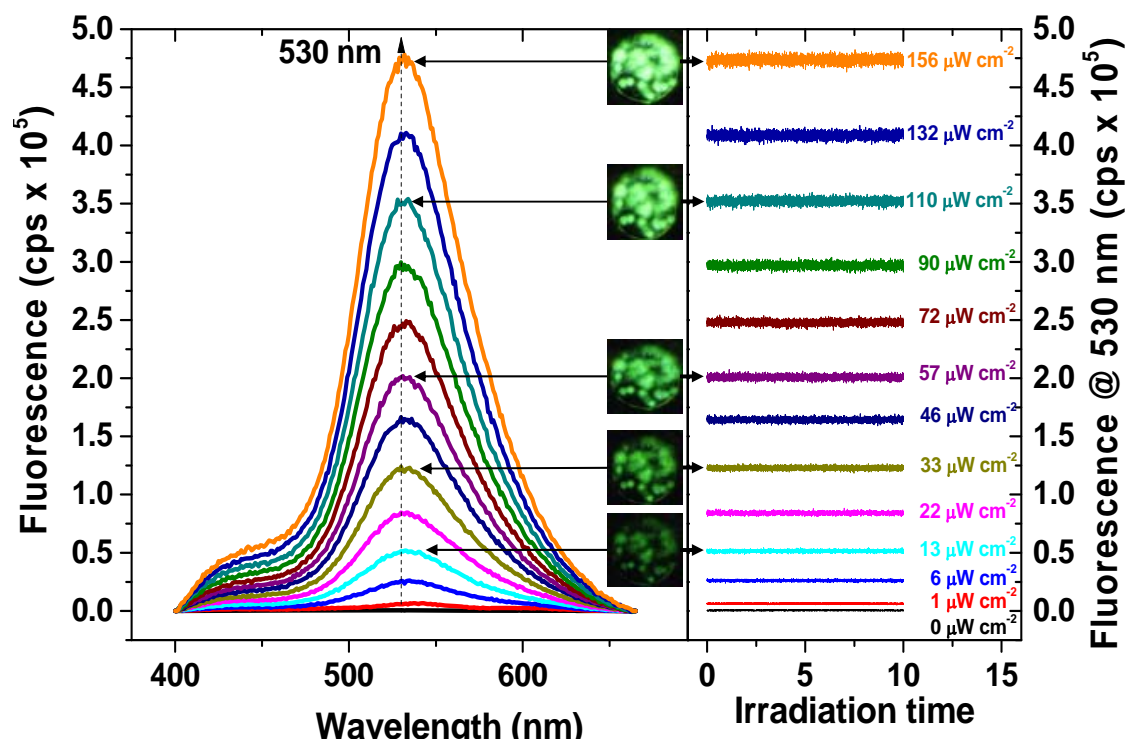

(e)

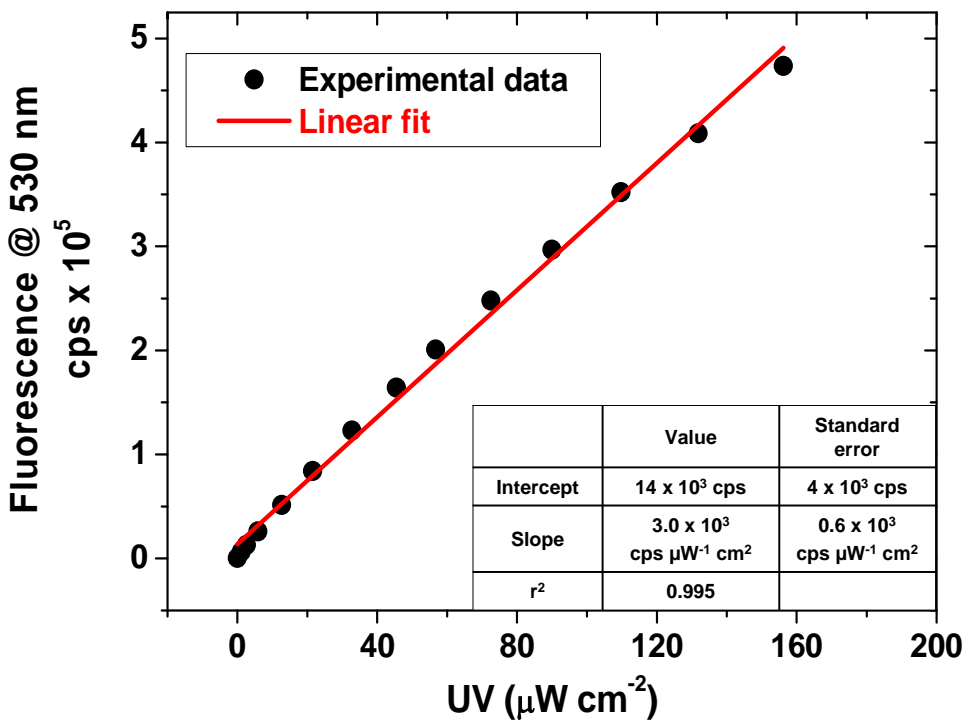

Fig. 8 Response towards UV illumination $\left(\lambda_{\mathrm{ex}}=350 \mathrm{~nm}\right.$ ) of 3-HF containing PAVD films. (a) Stability of the emission at $530 \mathrm{~nm}$ when the sample is illuminated during $6 \mathrm{~h}$ with UV light of $22 \mu \mathrm{W} \mathrm{cm} \mathrm{cm}^{-2}$. (b) Emission spectra as a function of the UV irradiance. Inserted photographs show the luminescent green emission for different values of UV irradiance. In these pictures the green zones correspond to each of the fibres that form the light guide system employed to illuminate the sample. (c) Stability of the emission intensity at $530 \mathrm{~nm}$ when the sample is illuminated during 10 min with different UV intensities. (d) Green emission recorded by a standard web cam for a PAVD film illuminated with solar light filtered by a $340 \mathrm{~nm}$ band pass and a $2.5 \%$ neutral filter. (e) Emission intensities as a function of the illumination irradiance.

\section{SUMMARY AND CONCLUSIONS}

Plasma polymer nanocomposite thin films containing 3-HF dye molecules have been synthesized by a solvent-less and one-step method. The films have been intended for 
their implementation as luminescent components in photonic devices. ToF-SIMS, XPS and FT-IR analyses indicate that the films consist of an organic macromolecular crosslinked structure incorporating entire molecules of the dye. FT-IR spectroscopy shows that the interaction between the plasma and the sublimated molecules regulates the structural characteristics of the polymeric matrix where the dye is trapped. Deposition parameters also control the amount of dye molecules in the films. This control over the composition and bond structures of the films (particularly the retention of $\pi$ bonds) allows for the deposition of organic thin films with rather high refractive index $(n=1.67$ at $632.8 \mathrm{~nm}$ ). Indeed, the optical properties can be finely adjusted within a wide range (i.e. $\Delta \mathrm{n} \sim 0.1$ at $\sim 632.8 \mathrm{~nm}$ ) for the suitable integration of the films in photonic structures. In this regard the transparency of the films in the visible region (e.g. $\mathrm{k}<2 \mathrm{x}$ $10^{-3}$ at $\lambda>550 \mathrm{~nm}$ ) together with its low surface roughness and stability are interesting properties. Fluorescence time resolved analyses indicate that 3-HF molecules are trapped in different chemical environments. In some of these environments the green emission is partially quenched. This also modifies the efficiency of the ESIPT process. These two factors affect the quantum yield of the trapped dye molecules, which changes from $\Phi=0.6 \%$ to $\Phi=3.7 \%$. According to this interpretation, the fluorescence efficiency of the films could be further enhanced by the fine adjustment of the chemical structures forming the plasma polymer host matrix. Moreover, the emission is noticeable even in ambient light conditions due to the high light absorption of these nanometric films and the large Stokes shift (i.e., absence of reabsorption). Finally, the use of the films as UV detectors has been successfully proved. After a pre-irradiation treatment, the fluorescence of the films is stable under a UV irradiation of $156 \mu \mathrm{W} \mathrm{cm}{ }^{-2}$ and the films have been shown to be able to detect very low UV light intensities below $10 \mu \mathrm{W} \mathrm{cm}$. This value is close to the detection limits of some commercial photodetectors.

\section{Acknowledgements}

We thank the Junta de Andalucía (Projects TEP5283 and TEP-8067) and the Ministry of Economy and Competitiveness (Projects CONSOLIDER CSD2008-00023, MAT201021228, and RECUPERA2020) for financial support.

1. A. Dodabalapur, Solid State Commun., 1997, 102, 259-267.

2. J. C. Goldschmidt, M. Peters, A. Bösch, H. Helmers, F. Dimroth, S. W. Glunz and G. Willeke, Sol. Energy Mater. Sol. Cells, 2009, 93, 176-182.

3. A. M. Adawi, M. M. Murshidy, P. W. Fry and D. G. Lidzey, ACS Nano, 2010, 4, 30393044.

4. R. A. S. Ferreira, P. S. André and L. D. Carlos, Opt. Mater., 2010, 32, 1397-1409.

5. H. Suzuki, A. Yokoo and M. Notomi, Polym. Adv. Technol., 2004, 15, 75-80.

6. F. J. Aparicio, M. Holgado, A. Borras, I. Blaszczyk-Lezak, A. Griol, C. A. Barrios, R. Casquel, F. J. Sanza, H. Sohlström, M. Antelius, A. R. González-Elipe and A. Barranco, Adv. Mater., 2011, 23, 761-765.

7. F. J. Aparicio, G. Lozano, I. Blaszczyk-Lezak, A. Barranco and H. Míguez, Chem. Mater., 2009, 22, 379-385.

8. F. J. Aparicio, I. Blaszczyk-Lezak, J. R. Sánchez-Valencia, M. Alcaire, J. C. González, C. Serra, A. R. González-Elipe and A. Barranco, J. Phys. Chem. C, 2012, 116, 8731-8740. 
9. I. Blaszczyk-Lezak, F. J. Aparicio, A. Borrás, A. Barranco, A. Álvarez-Herrero, M. Fernández-Rodríguez and A. R. González-Elipe, J. Phys. Chem. C, 2009, 113, 431-438.

10. F. J. Aparicio, A. Borras, I. Blaszczyk-Lezak, P. Gröning, A. Álvarez-Herrero, M. Fernández-Rodríguez, A. R. González-Elipe and A. Barranco, Plasma Processes and Polymers, 2009, 6, 17-26.

11. A. Barranco, F. Aparicio, A. Yanguas-Gil, P. Groening, J. Cotrino and A. R. González-Elipe, Chem. Vap. Deposition, 2007, 13, 319-325.

12. A. Barranco and P. Groening, Langmuir, 2006, 22, 6719-6722.

13. A. Barranco, M. Bielmann, R. Widmer and P. A. Groening, Adv. Eng. Mater., 2005, 7, 396-400.

14. H. Biederman, ed., Plasma Polymer Films, Imperial College Press, London, 2004.

15. R. d'Agostino, ed., Plasma Deposition, Treatment, and Etching of Polymers, Academic Press Inc., San Diego (US), 1990.

16. F. S. Denes and S. Manolache, Prog. Polym. Sci., 2004, 29, 815-885.

17. K. Ostrikov, E. C. Neyts and M. Meyyappan, Advances in Physics, 2013, 62, 113224.

18. A. Grill, Cold Plasma in Materials Fabrication, IEEE Press, Birmingham (GB), 1994.

19. J. Friedrich, Plasma Processes and Polymers, 2011, 8, 783-802.

20. G. P. Wells, I. C. Estrada-Raygoza, P. L. S. Thamban, C. T. Nelson, C.-W. Chung, L. J. Overzet and M. J. Goeckner, Plasma Processes and Polymers, 2013, 10, 119-135.

21. N. D. Boscher, D. Duday, P. Heier, K. Heinze, F. Hilt and P. Choquet, Surf. Coat. Technol., 2013, 234, 48-52.

22. K. D. Anderson, S. L. Young, H. Jiang, R. Jakubiak, T. J. Bunning, R. R. Naik and V. V. Tsukruk, Langmuir, 2011, 28, 1833-1845.

23. M. C. Vasudev, K. D. Anderson, T. J. Bunning, V. V. Tsukruk and R. R. Naik, ACS Appl. Mater. Interfaces, 2013, 5, 3983-3994.

24. S. Simovic, D. Losic and K. Vasilev, Chem. Commun., 2010, 46, 1317-1319.

25. W. Yan, Z. J. Han, B. T. Phung and K. Ostrikov, ACS Appl. Mater. Interfaces, 2012, 4, 2637-2642.

26. L. Martinu and D. Poitras, Journal of Vacuum Science \& Technology A: Vacuum, Surfaces, and Films, 2000, 18, 2619-2645.

27. W. Chen, L. Dai, H. Jiang and T. J. Bunning, Plasma Processes and Polymers, 2005, 2, 279-292.

28. L. G. Arnaut and S. J. Formosinho, Journal of Photochemistry and Photobiology A: Chemistry, 1993, 75, 1-20.

29. S. J. Formosinho and L. G. Arnaut, Journal of Photochemistry and Photobiology A: Chemistry, 1993, 75, 21-48.

30. P. K. Sengupta and M. Kasha, Chem. Phys. Lett., 1979, 68, 382-385.

31. D. McMorrow and M. Kasha, J. Phys. Chem., 1984, 88, 2235-2243.

32. A. J. G. Strandjord and P. F. Barbara, J. Phys. Chem., 1985, 89, 2355-2361.

33. P. K. Mandal and A. Samanta, J. Phys. Chem. A, 2003, 107, 6334-6339. 
34. M. Zayat, P. Garcia-Parejo and D. Levy, Chem. Soc. Rev., 2007, 36, 1270-1281.

35. S. Carturan, A. Quaranta, G. Maggioni, A. Vomiero, R. Ceccato and G. D. Mea, J. Sol-Gel Sci. Technol., 2003, 26, 931-935.

36. P. Chou, D. McMorrow, T. J. Aartsma and M. Kasha, J. Phys. Chem., 1984, 88, 4596-4599.

37. J. R. Dharia, K. F. Johnson and J. B. Schlenoff, Macromolecules, 1994, 27, 51675172.

38. S. Carturan, A. Quaranta, G. Maggioni, M. Bonafini and G. Della Mea, Sensors and Actuators A: Physical, 2004, 113, 288-292.

39. E. Monroy, F. Omnès and F. Calle, Semicond. Sci. Technol., 2003, 18, R33.

40. K. Liu, M. Sakurai and M. Aono, Sensors, 2010, 10, 8604-8634.

41. H. W. Lin, S. Y. Ku, H. C. Su, C. W. Huang, Y. T. Lin, K. T. Wong and C. C. Wu, Adv. Mater., 2005, 17, 2489-2493.

42. M. Alcaire, J. R. Sanchez-Valencia, F. J. Aparicio, Z. Saghi, J. C. Gonzalez-Gonzalez, A. Barranco, Y. O. Zian, A. R. Gonzalez-Elipe, P. Midgley, J. P. Espinos, P. Groening and A. Borras, Nanoscale, 2011, 3, 4554-4559.

43. Beamson G. and B. D., High Resolution XPS of Organic Polymers, Wiley\&Sons, Chichester, UK, 1992.

44. J. C. de Mello, H. F. Wittmann and R. H. Friend, Adv. Mater., 1997, 9, 230-232.

45. L. Porrès, A. Holland, L.-O. Pålsson, A. Monkman, C. Kemp and A. Beeby, Journal of Fluorescence, 2006, 16, 267-273.

46. H. Jiang, J. T. Grant, J. Enlow, W. Su and T. J. Bunning, J. Mater. Chem., 2009, 19, 2234-2239.

47. G. Maggioni, S. Carturan, A. Quaranta, A. Patelli, G. Della Mea and V. Rigato, Surf. Coat. Technol., 2003, 174-175, 1151-1158.

48. E. G. Lewars and R. E. March, Rapid Commun. Mass Spectrom., 2007, 21, 16691679.

49. A. Vavra, R. Linder and K. Kleinermanns, Chem. Phys. Lett., 2008, 463, 349-352.

50. M. Wang, T. Teslova, F. Xu, T. Spataru, J. R. Lombardi, R. L. Birke and M. Leona, J. Phys. Chem. C, 2007, 111, 3038-3043.

51. M. Buffa, S. Carturan, A. Quaranta, G. Maggioni and G. Della Mea, Opt. Mater., 2012, 34, 1219-1224.

52. G. Maggioni, S. Carturan, A. Quaranta, A. Patelli and G. Della Mea, Chem. Mater., 2002, 14, 4790-4795.

53. J. H. Looker and W. W. Hanneman, The Journal of Organic Chemistry, 1962, 27, 381-389.

54. G. Sokrates, Infrared Characteristic Group Frequencies: Tables and Charts, WileyInterscience, New York, 1994.

55. A. H. Kuptsov and G. N. Zhizhi, Handbook of Fourier transform Raman and infrared spectra of polymers, Elsevier, Amsterdam, 1998.

56. J. Ristein, R. T. Stief, L. Ley and W. Beyer, J. Appl. Phys., 1998, 84, 3836-3847. 
57. $\quad$ K. Tanaka, S. Nishio, Y. Matsuura and T. Yamabe, J. Appl. Phys., 1993, 73, 50175022.

58. K. D. Anderson, K. Marczewski, S. Singamaneni, J. M. Slocik, R. Jakubiak, R. R. Naik, T. J. Bunning and V. V. Tsukruk, ACS Appl. Mater. Interfaces, 2010, 2, 22692281.

59. V. I. Tomin and R. Jaworski, J. Mol. Struct., 2009, 924-926, 461-465.

60. H. Goktas, F. G. Ince, A. Iscan, I. Yildiz, M. Kurt and I. Kaya, Synth. Met., 2009, 159, 2001-2008.

61. M. C. Kim, S. H. Cho, J. G. Han, B. Y. Hong, Y. J. Kim, S. H. Yang and J. H. Boo, Surf. Coat. Technol., 2003, 169-170, 595-599.

62. P. G. Parejo, M. Zayat and D. Levy, J. Mater. Chem., 2006, 16, 2165-2169.

63. J.-g. Liu and M. Ueda, J. Mater. Chem., 2009, 19, 8907-8919.

64. T. Hanemann, in SPIE Newsroom, 2008.

65. T. Sato, K. Moriki and M. Yumoto, IEEJ Transactions on Electrical and Electronic Engineering, 2010, 5, 416-421.

66. J. O. Enlow, H. Jiang, J. T. Grant, K. Eyink, W. Su and T. J. Bunning, Polymer, 2008, 49, 4042-4045.

67. H. Jiang, K. O'Neill, J. T. Grant, S. Tullis, K. Eyink, W. E. Johnson, P. Fleitz and T. J. Bunning, Chem. Mater., 2004, 16, 1292-1297.

68. F. F. Shi, Surf. Coat. Technol., 1996, 82, 1-15.

69. S. Basu, S. Mondal and D. Mandal, J. Chem. Phys., 2010, 132, 034701.

70. A. S. Klymchenko, V. G. Pivovarenko and A. P. Demchenko, Spectrochimica Acta Part A: Molecular and Biomolecular Spectroscopy, 2003, 59, 787-792.

71. C. Bringer, V. Bardinal, E. Daran, T. Camps, Y. G. Boucher, G. Almuruneau, O. Gauthier-Lafaye, P. Dubreuila, J. B. Doucet and C. Fontaine, Micro-Optics, Vcsels, and Photonic Interconnects, 2004, 5453, 209-216.

72. R. d'Agostino, P. Favia, C. Oehr and M. R. Wertheimer, Plasma Processes and Polymers, 2005, 2, 7-15.

73. A. Quaranta, S. Carturan, G. Maggioni, R. Ceccato and G. Della Mea, J. Non-Cryst. Solids, 2003, 322, 1-6. 